\title{
The Solar Probe Plus Mission: Humanity's First Visit to Our Star
}

\author{
N.J. Fox ${ }^{1}$ - M.C. Velli ${ }^{2}$ S.D. Bale ${ }^{3}$ - R. Decker ${ }^{1}$ A. Driesman ${ }^{1} \cdot$ R.A. Howard ${ }^{4}$. \\ J.C. Kasper ${ }^{5,6}$ • J. Kinnison ${ }^{1}$ - M. Kusterer ${ }^{1}$ - D. Lario ${ }^{1}$ - M.K. Lockwood ${ }^{1}$ \\ D.J. McComas ${ }^{7,8}$ • N.E. Raouafi ${ }^{1}$ A. Azabo ${ }^{9}$
}

Received: 10 December 2014 / Accepted: 14 October 2015 / Published online: 11 November 2015

(C) The Author(s) 2015. This article is published with open access at Springerlink.com

\begin{abstract}
Solar Probe Plus (SPP) will be the first spacecraft to fly into the low solar corona. SPP's main science goal is to determine the structure and dynamics of the Sun's coronal magnetic field, understand how the solar corona and wind are heated and accelerated, and determine what processes accelerate energetic particles. Understanding these fundamental phenomena has been a top-priority science goal for over five decades, dating back to the 1958 Simpson Committee Report. The scale and concept of such a mission has been revised at intervals since that time, yet the core has always been a close encounter with the Sun. The mission design and the technology and engineering developments enable SPP to meet its science objectives to: (1) Trace the flow of energy that heats and accelerates the solar corona and solar wind; (2) Determine the structure and dynamics of the plasma and magnetic fields at the sources of the solar wind; and (3) Explore mechanisms that accelerate and transport energetic particles. The SPP mission was confirmed in March 2014 and is under development as a part of NASA's Living with a Star (LWS) Program. SPP is scheduled for launch in mid-2018, and will perform 24 orbits over a 7-year nominal mission duration. Seven Venus gravity assists gradually reduce SPP's perihelion from 35 solar radii $\left(R_{S}\right)$ for the first orbit to $<10 R_{S}$ for the final three orbits. In this paper we present the science,
\end{abstract}

N.J. Fox

Nicola.Fox@jhuapl.edu

1 Johns Hopkins University Applied Physics Laboratory, Laurel, MD 20723, USA

2 Jet Propulsion Laboratory, California Institute of Technology, Pasadena, CA 91109, USA

3 University of California at Berkeley, Berkeley, CA 94720, USA

4 Space Science Division, Naval Research Laboratory, Washington, DC 20375, USA

5 University of Michigan, Ann Arbor, MI 48109, USA

6 Harvard-Smithsonian Center for Astrophysics, Cambridge, MA 02138, USA

7 Southwest Research Institute, San Antonio, TX 78228, USA

8 University of Texas at San Antonio, San Antonio, TX 78249, USA

9 Goddard Space Flight Center, Greenbelt, MD 20771, USA 
mission concept and the baseline vehicle for SPP, and examine how the mission will address the key science questions

Keywords Solar Probe Plus · SPP · Corona · Heliophysics · NASA mission · Solar wind

\section{Introduction}

Solar Probe Plus (SPP) will sample the solar corona to reveal how it is heated and the solar wind and solar energetic particles are accelerated. Solving these problems has been a top science goal for over 50 years (see Box 1). During the seven-year mission, seven Venus gravity assist (VGA) maneuvers will gradually lower the perihelia to $<10 R_{S}$, the closest any spacecraft has come to the Sun. Throughout the 7-year nominal mission duration, the spacecraft will spend a total of 937 hours inside $20 R_{S}, 440$ hours inside $15 R_{S}$, and 14 hours inside $10 R_{S}$, sampling the solar wind in all its modalities (slow, fast, and transient) as it evolves with rising solar activity toward an increasingly complex structure. SPP will orbit the Sun in the ecliptic plane, and so will not sample the fast wind directly above the Sun's polar regions (see Fig. 1). However, the current mission design (Lockwood et al. 2012) compensates for the lack of in-situ measurements of the fast wind above the polar regions by the relatively long time SPP spends inside $20 R_{S}$. This will allow extended measurement of the equatorial extensions of high-latitude coronal holes and equatorial coronal holes. At a helioradius $\approx 35 R_{S}$, there are two periods per orbit (one inbound and one outbound) when SPP will be in quasi-corotation with the Sun and will cross a given longitudinal sector slowly. In these intervals, known as fast radial scans, the spacecraft will sample the solar wind over large radial distances within a given flux tube before moving across the sector. These measurements will yield additional information on the spatial/temporal dependence of structures in the solar wind and on how they merge in the inner heliosphere. This paper describes the science, mission concept, and reference vehicle for the SPP mission.

\section{Science Overview}

The SPP mission targets processes and dynamics that characterize the Sun's expanding corona and solar wind. SPP will explore the inner region of the heliosphere through insitu and remote sensing observations of the magnetic field, plasma, and energetic particles. The solar magnetic field plays a defining role in forming and structuring the solar corona and the heliosphere. In the corona, closed magnetic field lines confine the hot plasma in loops, while open magnetic field lines guide the solar wind expansion in the inner corona. The energy that heats the corona and drives the wind derives from photospheric motions, and is channeled, stored, and dissipated by the magnetic fields that emerge from the convection zone and expand in the corona where they dominate almost all physical processes therein. Examples of these are waves and instabilities, magnetic reconnection, and turbulence, which operate on a vast range of spatial and temporal scales. Magnetic fields play also a critical role in coronal heating and solar wind acceleration. They are conduits for waves, store energy, and propel plasma into the heliosphere through complex forms of magnetic activity (e.g., coronal mass ejections (CMEs), flares, and small-scale features such as spicules and jets). How solar convective energy couples to magnetic fields to produce the multifaceted heliosphere is central to SPP science. 


\section{Box 1. A Brief History of Solar Probe}

Recommendations for a solar probe mission date back to the October 1958 National Research Council's (NRC) Space Studies Board recommendation for a future mission to send a spacecraft inside the orbit of Mercury to measure the particles and fields environment near the Sun. These recommendations have continued for over five decades, including the identification of Solar Probe as a top priority in the 2003 NRC Decadal survey (Solar and Space Physics Survey Committee, The Sun to the Earth - and Beyond: A Decadal Research Strategy in Solar and Space Physics, National Academy Press, Washington, DC, 2003), and are re-affirmed in the 2013 NRC Decadal survey (Solar and Space Physics: A Science for a Technological Society. Washington, DC: The National Academies Press, 2013). Beginning in 1962, eight major science and implementation studies were completed (McComas et al., 2007), culminating in the 2008 SPP Science and Technology Definition Team (STDT) report (McComas et al., 2008) and supporting SPP Mission Engineering Study Report (JHUAPL, prepared under contract NNN06AA01C, Laurel, MD, 2008).
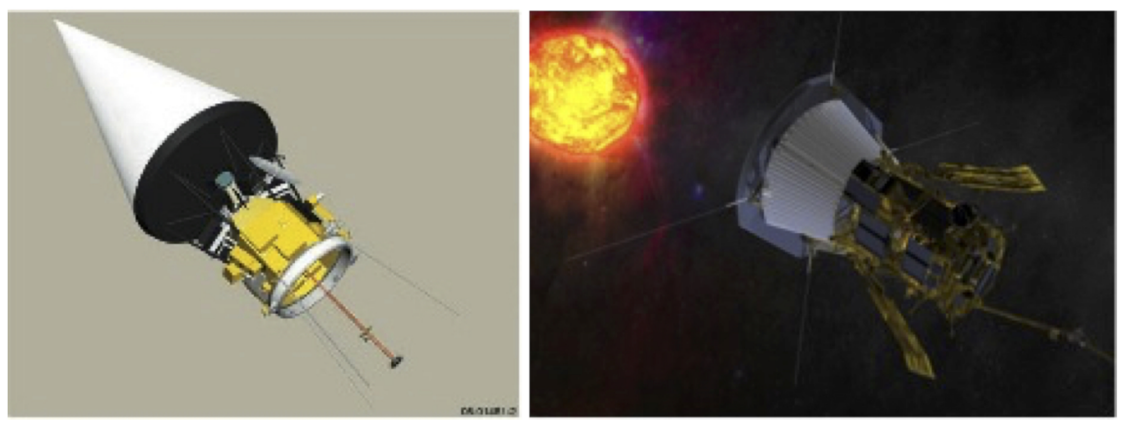

The SPP mission described in this paper (right-hand figure) has a very different mission design from those envisioned in previous studies (e.g., Solar Probe, 2005 design [SP2005], left-hand figure), although the primary science goal remains the same. The earlier missions involved one or two flybys of the Sun at a perihelion distance of $\approx 4 R_{S}$ by a spacecraft placed into a solar polar orbit using a Jupiter gravity assist. In contrast, SPP will make many nearSun passes at increasingly lower perihelia, confined to within a few degrees of the ecliptic plane and with a closest approach farther from the Sun $\left(<10 R_{S}\right)$. An extensive study was conducted (McComas et al., 2008) to assess the impact of the changed mission design on Solar Probe science. The study determined that the new concept would allow all of the scientific goals to be attained and, in fact, presented a number of significant advantages over the old design, largely because of the far larger number of passes and much greater data sampling in this region (i.e., 24 orbits instead of 2 orbits and over 2100 hours instead of $\sim 160$ hours inside of $\left.30 R_{S}\right)$. Moreover, at closest approach, the lower speed $\left(195 \mathrm{~km} \mathrm{~s}^{-1}\right)$ of SPP compared to that of SP2005 $\left(308 \mathrm{~km} \mathrm{~s}^{-1}\right)$ will allow better sampling of the fast solar wind structures, such as plumes.

At times of low solar activity (i.e., solar cycle minima), the solar wind is bimodal. There is a dominant quasi-steady high-speed wind that originates in open-field polar coronal holes and a variable, low-speed wind that originates around the equatorial streamer belt (McComas et al. 1998, Fig. 1a, c). As solar activity increases and evolves toward solar cycle maxima, this orderly bimodal configuration of the corona and solar wind breaks down. Polar holes shrink, and the heliospheric current sheet becomes warped due to magnetic flux emergence whose coronal manifestations are active regions, equatorial coronal holes, and coronal streamers at higher heliographic latitudes. A mixture of fast flows from smaller 
coronal holes and transients, embedded in a slow-to-moderate-speed wind, appears at all latitudes (e.g., McComas et al. 2003, Fig. 1b).

SPP's closest approach to the Sun ( $<10 R_{S}$ from Sun center) will enable it to measure coronal conditions leading to the nascent solar wind and eruptive transients that create space weather. The seven-year prime mission will permit observations over a significant portion ( $>60 \%$ ) of a solar cycle. Direct plasma, magnetic field, and energetic particle measurements will allow testing of and discrimination among a broad range of theories and models that describe the Sun's coronal magnetic field, the heating and acceleration of the solar wind and energetic particle acceleration.

The primary science objective of the SPP mission is to determine the structure and $d y$ namics of the Sun's coronal magnetic field and to understand how the corona is heated, the solar wind accelerated, and how energetic particles are produced and their distributions evolve. To advance the scientific knowledge needed to characterize the inner heliosphere, the SPP mission has defined the following three overarching science objectives.

\section{Trace the flow of energy that heats the solar corona and accelerates the solar} wind.

1a. How is energy from the lower solar atmosphere transferred to, and dissipated in, the corona and solar wind?

1b. What processes shape the non-equilibrium velocity distributions observed throughout the heliosphere?

1c. How do the processes in the corona affect the properties of the solar wind in the heliosphere?

2. Determine the structure and dynamics of the plasma and magnetic fields at the sources of the solar wind.

2a. How does the magnetic field in the solar wind source regions connect to the photosphere and the heliosphere?

2b. Are the sources of the solar wind steady or intermittent?

2c. How do the observed structures in the corona evolve into the solar wind?

3. Explore mechanisms that accelerate and transport energetic particles.

3a. What are the roles of shocks, reconnection, waves, and turbulence in the acceleration of energetic particles?

3b. What are the source populations and physical conditions necessary for energetic particle acceleration?

3c. How are energetic particles transported in the corona and heliosphere?

SPP will make in-situ and remote measurements from $<10 R_{S}$ to at least $0.25 \mathrm{AU}$ $\left(53.7 R_{S}\right)$. Measurements of the region where the solar wind originates and where the most hazardous solar energetic particles are energized will improve our ability to characterize and forecast the radiation environment of the inner heliosphere. SPP will measure local particle distribution functions, density and velocity field fluctuations, and electromagnetic fields within $0.25 \mathrm{AU}$ of the Sun. These data will help answer the basic questions of how the solar corona is powered, how the energy is channeled into the kinetics of particle distribution functions in the solar corona and wind, and how such processes relate to the turbulence and wave-particle dynamics observed in the heliosphere. Cross-correlation of velocity, density, and electromagnetic fluctuations will allow a partial separation of spatial and temporal effects.

The physical conditions of the region below $20 R_{S}$ are important in determining largescale properties such as solar wind angular momentum loss and global heliospheric struc- 


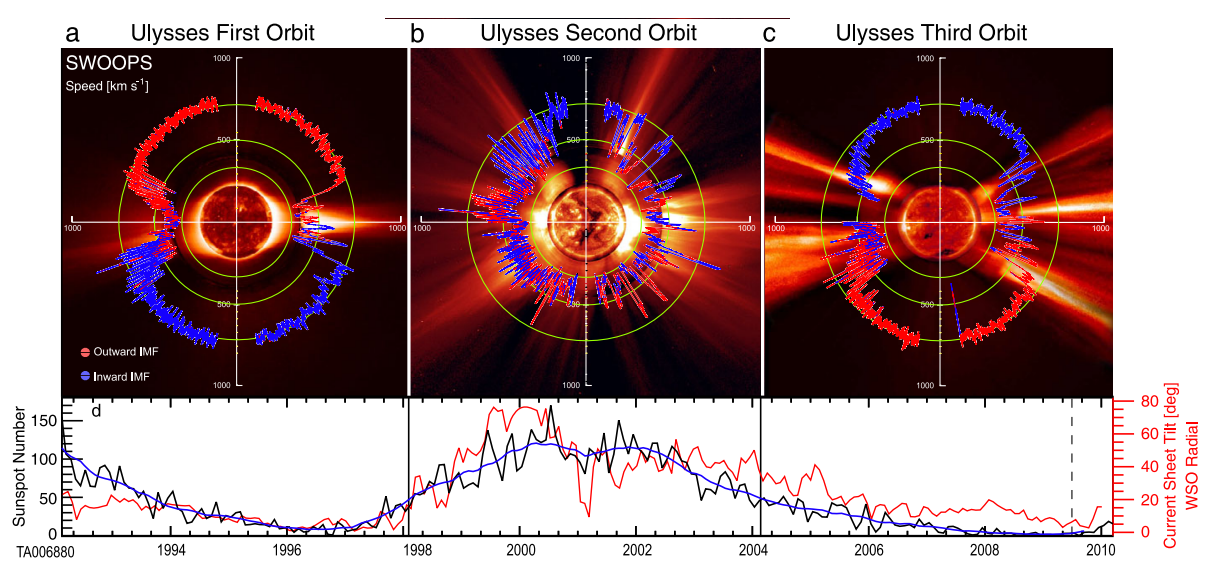

Fig. 1 Solar wind speed as a function of heliographic latitude illustrating the relationship between the structure of the solar wind and coronal structure at solar minimum $(\mathbf{a}, \mathbf{c})$ and solar maximum $(\mathbf{b})$. Ulysses SWOOPS solar wind data are superposed on composite solar images obtained with the SOHO EIT and LASCO C2 instruments and with the Mauna Loa K-coronameter. (d) Solar cycle evolution. Adopted from McComas et al. (2003)

ture. The Alfvénic critical surface, where the solar wind speed overtakes the Alfvén speed, is believed to lie in this region (e.g., Katsikas et al. 2010; Goelzer et al. 2014). This surface defines the point beyond which the plasma ceases to corotate with the Sun, i.e., where the magnetic field loses its rigidity to the plasma. In this region solar wind physics changes because of the multi-directionality of wave propagation (waves moving sunward and antisunward can affect the local dynamics including the turbulent evolution, heating and acceleration of the plasma). This is also the region where velocity gradients between the fast and slow speed streams develop, forming the initial conditions for the formation, further out, of corotating interaction regions (CIRs). In the remainder of this section, we summarize SPP science questions and discuss how the mission design will enable investigators to address these questions.

\subsection{Trace the Flow of Energy that Heats the Solar Corona and Accelerates the Solar Wind}

\subsubsection{How is Energy from the Lower Solar Atmosphere Transferred to, and Dissipated in, the Corona and Solar Wind?}

How convective kinetic energy is transferred and dissipated to generate the hot corona and solar wind are fundamental open questions in heliophysics and astrophysics as a whole. The turbulent motion in the convection zone below the photosphere is a sufficient mechanical energy reservoir to power the corona and heliosphere (e.g., convective hydrodynamic buffeting, shock formation, waves). Substantial energy may be stored in complex topologies of coronal magnetic fields. Parker (1972) conjectured that such magnetic field stresses induced by the "tangling" of magnetic field lines lead to the formation of unstable current sheets in the corona whose dissipation via "nanoflares" may provide sufficient energy to heat the corona (see also Parker 1991). Numerical simulations show that the macroscopic heating rate depends on the dimension of closed loops, the intensity of the magnetic field and a well-defined, turbulence-associated coronal oscillation spectrum (e.g., Rappazzo and 


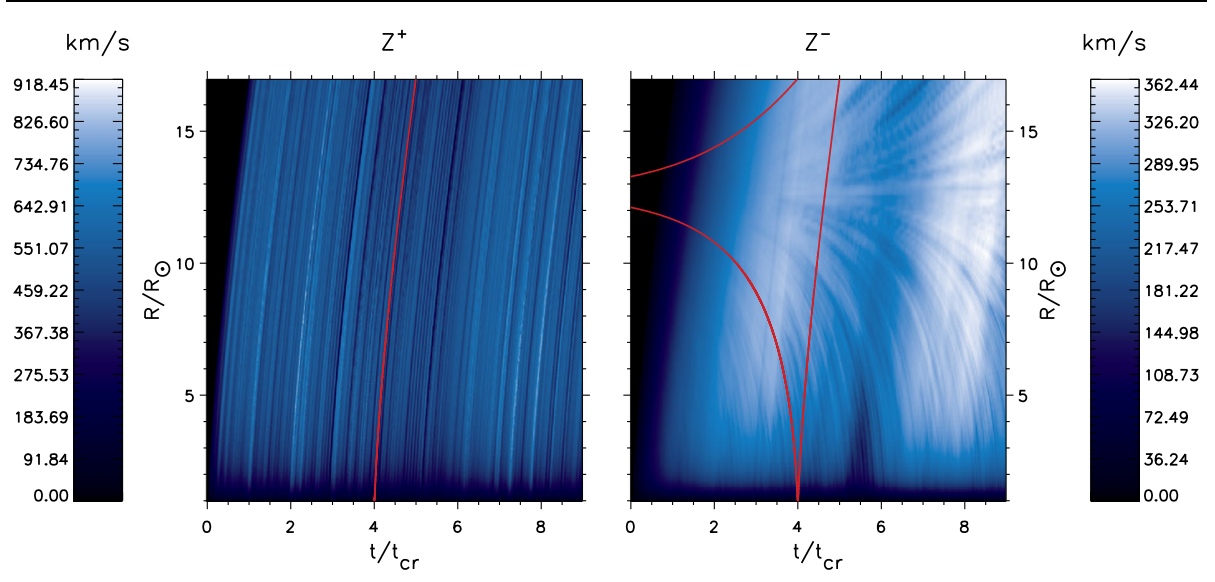

Fig. 2 SPP will measure the turbulence levels inside the critical point, verifying whether a nonlinear cascade contributes to coronal heating. Contour plots of the energy expressed in terms of Elsässer variables $\left(Z^{ \pm}\right)$in outward $\left(Z^{+}\right)$and inward $\left(Z^{-}\right)$propagating Alfvén waves as a function of time and distance. $t_{c r}$ is the Alfvén critical time. Wave nonlinear evolution is due to wave reflection at the Alfvén critical height $\left(\sim 12-13 R_{S}\right)$, which seems to be a source of outward and inward waves beyond which all waves propagate outward. Adopted from Verdini et al. (2009)

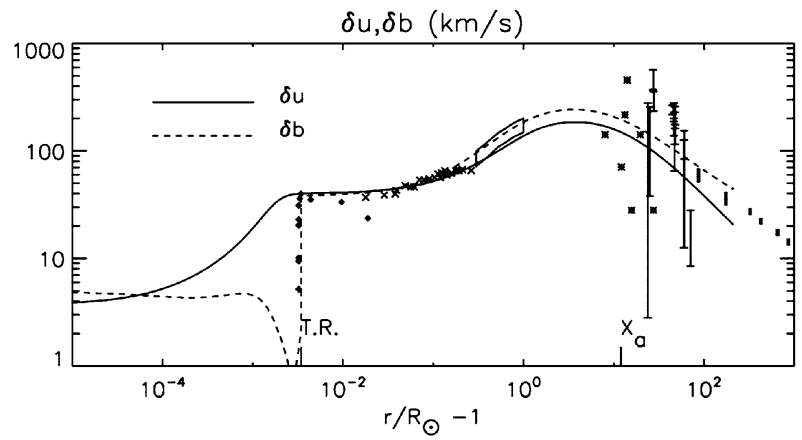

Fig. 3 SPP measurements of the rms energies inside the Alfvén point $\left(\mathrm{X}_{\mathrm{a}}\right.$ in figure) will support or disprove low-frequency Alfvénic turbulence as a contributor to fast solar wind heating and acceleration. The $r m s$ amplitudes of solar wind velocity $(\delta u)$ and magnetic field ( $\delta b$ in velocity units) are shown functions of heliocentric distance for a photospheric Kolmogorov spectrum with $40 \mathrm{~km} \mathrm{~s}^{-1}$. Adopted from Verdini and Velli (2007)

Parker 2013; Velli 2010; Asgari-Targhi et al. 2013). Axford and McKenzie (1992) suggested that high-frequency waves generated by such reconnection events in the upper chromosphere/transition region would heat minor ions in coronal holes via ion-cyclotron resonance. Alternatively, the main heating could come from a reflection-generated nonlinear cascade of lower frequency Alfvén waves - the open field counterpart of the closed field heating model discussed above (Velli et al. 1989; Matthaeus et al. 1999; Verdini and Velli 2007; Cranmer et al. 2007; Verdini et al. 2010; Perez and Chandran 2013; Lionello et al. 2014). Such incompressible turbulence models of coronal heating appear to be successful in creating realistic high-speed solar wind streams (Cranmer 2010; Verdini et al. 2010, Figs. 2, 3), though the role of the Alfvénic turbulence observed in-situ, and whether it is a remnant of heating and accelerating flux, are unclear (Roberts 2010). A further heating mechanism, so-called 
stochastic heating, relies on a turbulence fluctuation field to drive perpendicular proton and minor ion temperatures higher (Xia et al. 2013). Alfvén waves propagating from the corona could also drive compressive interactions and steepen to form shocks, either directly or indirectly through decay instability or mode conversion (Malara and Velli 1996; Malara et al. 1997). Coronal heating and acceleration models based solely on this effect have been developed recently (Suzuki and Inutsuka 2006) leading to a solar wind which contains extremely large fluctuations in radial velocity and density close to the Sun. Such density oscillations along a specific flux bundle of field lines should be readily observable by SPP. In particular, during the fast radial scans (when the distance to the Sun increases or decreases rapidly) and at perihelion, increases in the variability of the flow, in the presence of steepened Alfvénic wave-fronts and shocks, should be observed.

SPP measurements of the local plasma and wind conditions, during the fast radial scans and at perihelia, in conjunction with other remote sensing coronal observations (e.g., Solar Orbiter, ground-based, etc.) will provide a quantitative verification of solar wind acceleration scaling laws (e.g., Schwadron and McComas 2003). Statistical properties of energy emission events and the relative heating of ions and electrons in nanoflares will be compared with the statistics of radio signatures of impulsive events measured at SPP. SPP will measure plasma and fields in situ, enabling evaluation of the local density conditions and sensing the radio signals of energy bursts in the corona. These data will enable us to quantify different energy transport and conversion mechanisms and test theories for coronal heating and solar wind acceleration. SPP will characterize the turbulence and energy budgets and their evolution along the orbits, compare with sources at the Sun and along the trajectory of the spacecraft, and search for signatures of candidate dissipation mechanisms at kinetic scales.

\subsubsection{What Processes Shape the Non-equilibrium Velocity Distributions Observed Throughout the Heliosphere?}

Observed electron velocity distribution functions (eVDFs) exhibit non-Maxwellian characteristics for all wind types (see Fig. 4). The eVDFs permanently exhibit three different components: a thermal core and a supra-thermal halo, which are present at all pitch angles, and a sharply magnetic field aligned "strahl" which is usually propagating antisunward (Rosenbauer et al. 1977; Maksimovic et al. 2005). It has been suggested that significant contributions to coronal heating come from energy stored in non-thermal wings of particle distribution functions and that higher temperatures and subsequent outflows would then arise naturally through velocity filtration (Scudder 1994; Maksimovic et al. 1997; Zouganelis et al. 2004). On the other hand, proton velocity distribution functions (pVDFs) measured in the inner heliosphere, especially in the fast wind streams, typically have anisotropic cores that are well represented by bi-Maxwellian distributions, with $T_{\perp}>T_{\|}$, where $T_{\|}$and $T_{\perp}$ are temperatures parallel to and perpendicular to the magnetic field, respectively. These VDFs are constrained by wave-particle interactions via plasma instabilities (Kasper et al. 2002, 2013; Hellinger et al. 2006; Matteini et al. 2007, 2012, 2013; Bale et al. 2009, see Fig. 5). They also exhibit a field-aligned beam component that flows ahead of the core by about the local Alfvén speed. Alpha particles share similar properties but also flow systematically with a higher speed up to about the Alfvén speed, compared to the protons. The temperature anisotropy is remarkable in that, for an expanding solar wind, without perpendicular heating, magnetic moment conservation would favor higher parallel temperatures. Identifying the kinetic processes which create these distinctive properties is essential to understanding the solar wind. 

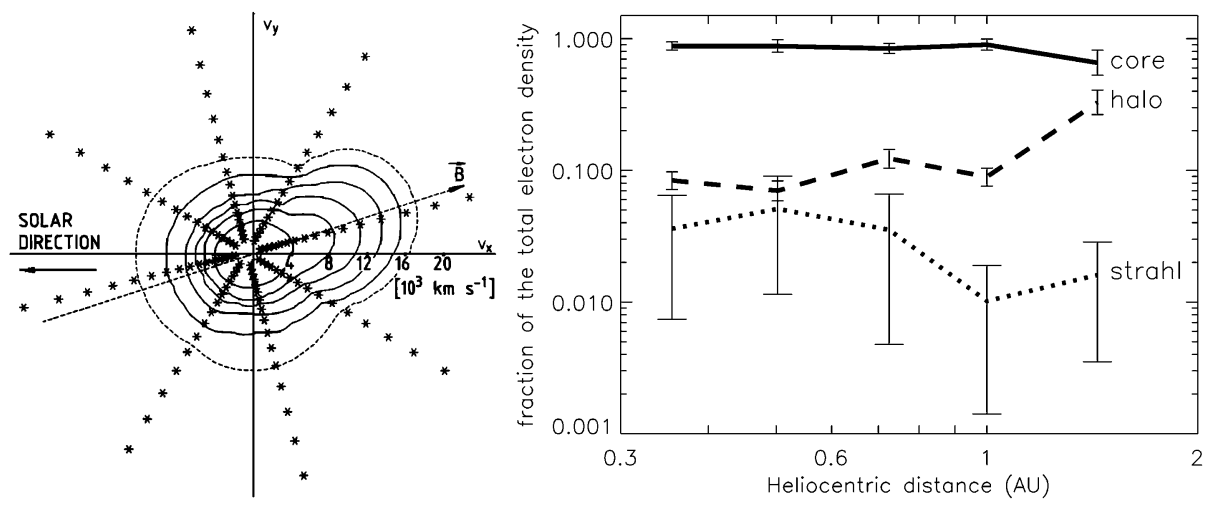

Fig. 4 SPP will measure the different parts of the electron distribution function down to $9.86 R_{S}$, determining the dynamic relevance of electrons in solar wind acceleration. (Left; from Pilipp et al. 1987) Electron velocity distribution function in the solar wind as measured by the plasma instrument on the Helios spacecraft at 1 AU. Note the distinct bulge along the mean magnetic field direction, which is the so-called strahl, a suprathermal population carrying the heat flux, together with the halo, the hotter isotropic component which is slightly displaced with respect to the maximum of the core part. (Right; from Maksimovic et al. 2005) Radial decline (increase) of the number of strahl (halo) electrons with heliocentric distance as measured by Helios, WIND and Ulysses

SPP will provide detailed 3D VDFs of electrons, protons, alphas and measurements of the 3D magnetic field vector to determine angular and temperature anisotropies as a function of helioradius and in different solar wind conditions. In conjunction with measurement of the multi-component electromagnetic spectrum and resulting spectral indices from the inertial range to the gyro-scale frequencies, SPP will identify the kinetic regulation processes and discriminate among various heating and dissipation mechanisms. SPP will determine the helioradii at which instability thresholds due to anisotropies are reached and whether ion beam formation is an evolutionary heliospheric process or originates from processes low in the corona or both.

\subsubsection{How do the Processes in the Corona Affect the Properties of the Solar Wind in the Heliosphere?}

Figure 6 shows power-law frequency spectra, with break points (blue and red dots) that separate steepening power-laws representing the different stages of Alfvénic turbulent cascade. The break points move to lower frequencies with increasing helioradius. Thus, turbulence in fast solar wind streams and also in slower streams that are far from the plasma and current sheets are dominated by Alfvénic fluctuations (Bruno and Carbone 2005, 2013; Telloni et al. 2015). This helioradial evolution is evidence for an ongoing non-linear cascade with heating rates estimated to be in the range required to explain the in-situ heating of the solar wind (Vasquez et al. 2007). However, the overall way in which energy per unit mass $(E)$ in the fluctuations decays with distance $\left(E \sim R^{-1.1}\right)$ is very close to that predicted based on solar wind expansion effects alone $\left(E \sim R^{-1}\right)$. The good correlation of Alfvénic periods with high-speed streams mentioned above (Matteini et al. 2014) might be taken as indirect evidence for the Alfvén wave acceleration of high-speed streams, but is the energy flux in Alfvén waves at the Sun sufficient to produce the acceleration? Roberts $(1989,2010)$ claim that the flux is insufficient, based on a linear scaling of the energies backwards. There is strong evidence that the nonlinear cascade is anisotropic, but it is not known how the 

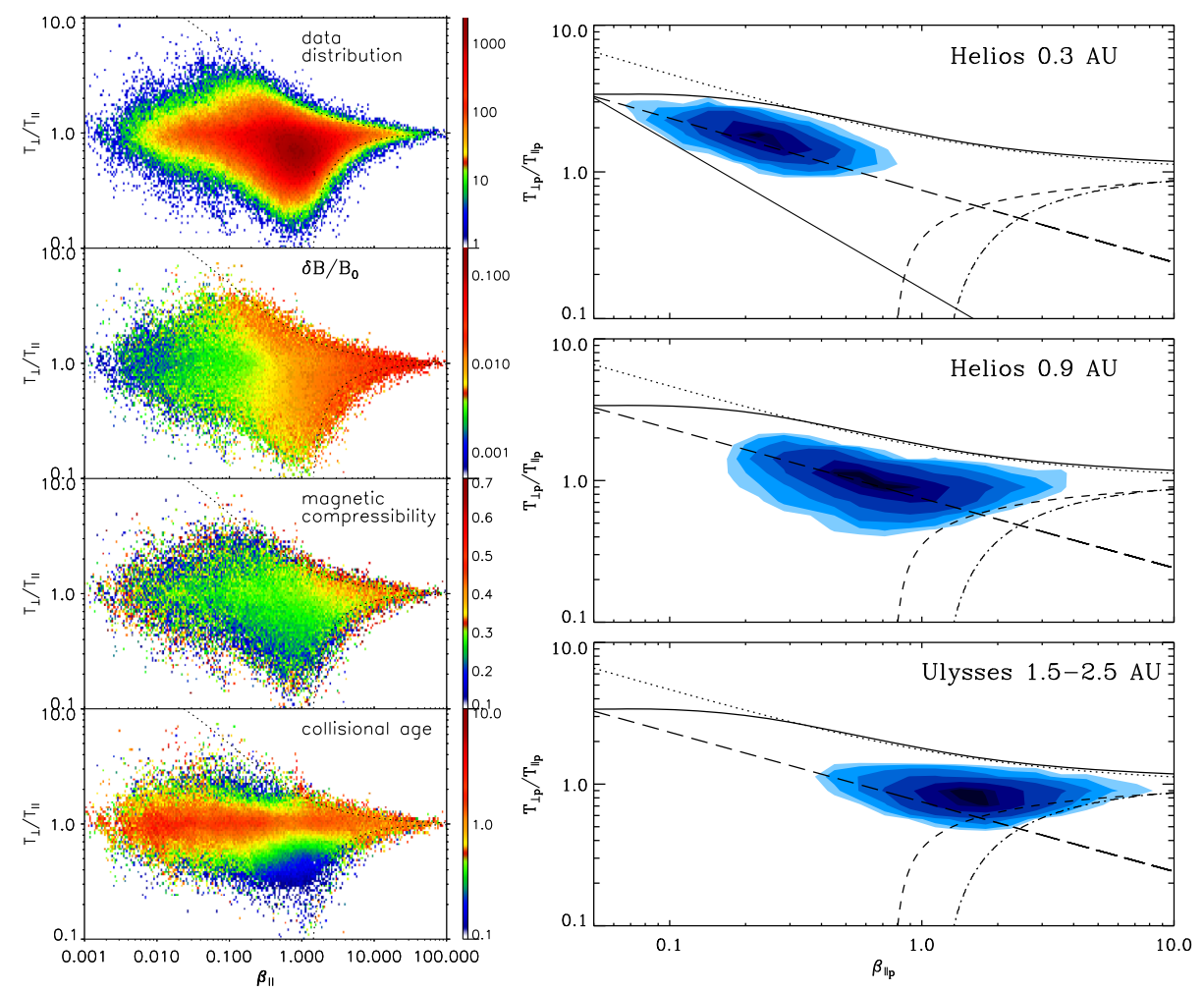

Fig. 5 SPP will measure ion and electron velocity distribution functions and corresponding plasma instabilities in the very low beta conditions of the coronal source, clarifying the different mechanisms responsible for the evolution of ion velocity distribution functions. (Left; from Bale et al. 2009) 1 AU slow solar wind data from the WIND spacecraft. The different plasma parameters (i.e., temperature anisotropy (upper panel), magnetic field fluctuation amplitude $\delta B / B_{0}$, the magnetic compressibility, and the collisional age) are plotted for different temperature anisotropy as a function of the plasma $\beta_{\|}$. The data show that $T_{\perp} / T_{\|}$(upper panel) is constrained by the oblique proton mirror and firehose instability thresholds (upper and lower dotted lines, respectively), $\delta B / B_{0}$ is enhanced along the instability thresholds, and the "collisional age" is largest where parallel and perpendicular temperatures are identical. The magnetic compressibility does not show any particular trend. (Right; from Matteini et al. 2007) Simulated temperature anisotropy of the fast solar wind protons as a function of $\beta_{\|}$at different heliocentric distances

anisotropy is generated and evolves (Horbury et al. 2008; Podesta 2009). Slow-fast wind shears, fine-scale structures, and gradients are all candidate mechanisms (Tu and Marsch 1990; Breech et al. 2008). To determine how the plasma environment affects the dynamical evolution of solar wind turbulence it is essential to measure the plasma and magnetic field fluctuations in the solar wind as close to the Sun as possible, before the effects of mechanisms such as velocity shear become significant, and then to observe how the turbulence evolves with heliocentric distance.

SPP will address these phenomena using measurements of the fluctuation energies and their spectra along its trajectory. Measurements of the plasma velocity and magnetic field fluctuations at varying distance from the Sun (specifically during the fast radial scans and perihelion encounters, where some distinction between spatial structures can be made) will unequivocally determine whether and to what extent the currently observed fluctuations are remnants of coronal processes, and how observed flux structures are connected to coronal 

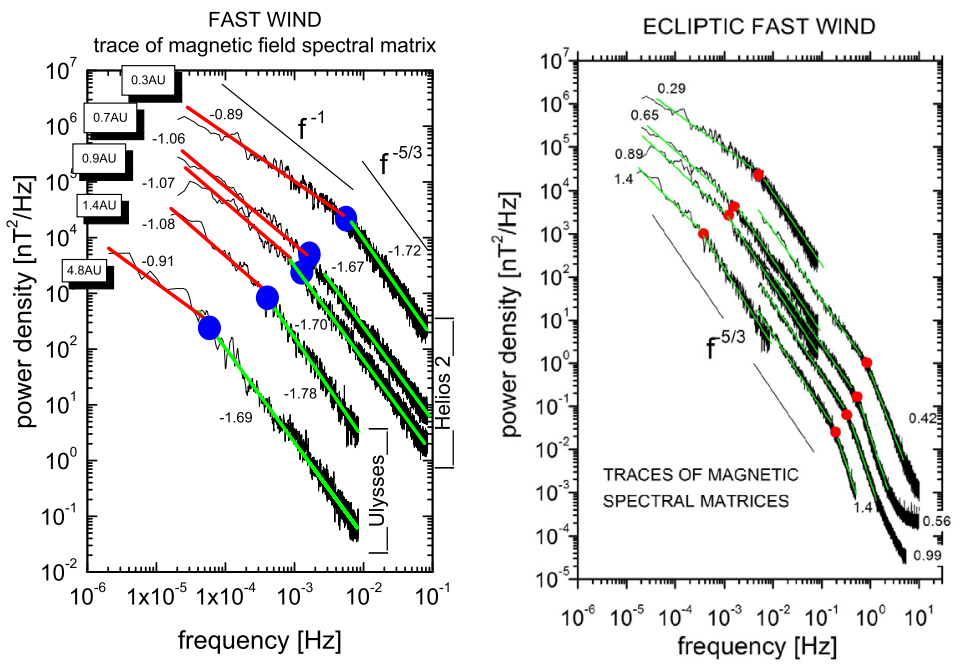

Fig. 6 SPP will verify changes in spectral shape and total energy of power density spectra of magnetic field fluctuations along its orbit, allowing us to confirm or disprove the role of Alfvén waves in fast solar wind acceleration. (Left; from Bruno and Carbone 2013) Power density spectra of magnetic field fluctuations as a function of heliodistance measured by Helios 2 and Ulysses. (Right; from Telloni et al. 2015) Power density spectra extended to higher frequencies using data from Helios 2, Ulysses, Wind, and MESSENGER data. The spectral break (blue and red dots) shown by each spectrum moves to lower and lower frequency with increasing heliodistance

sources. SPP will directly probe the critical region around the Alfvén point. The source of very low frequency variability and of non-Gaussian probability distributions of field strengths and energy density (Marsch and Tu 1994, 1997) as well as " $1 / f$ noise" will be established.

\subsection{Determine the Structure and Dynamics of the Plasma and Magnetic Fields at the Sources of the Solar Wind}

Several plasma physical processes-waves and instabilities, magnetic reconnection, turbulence-operating on a large range of spatial and temporal scales are believed to play key roles in coronal heating and solar wind acceleration. Measurements made below the Alfvén point will enable SPP to determine if the sources of the solar wind are continuous or intermittent and if these sources merge to form the solar wind only at higher radial distances.

\subsubsection{How Does the Magnetic Field in the Solar Wind Source Regions Connect to the Photosphere and the Heliosphere?}

Potential Field Source Surface (PFSS; Altschuler and Newkirk 1969; Schatten et al. 1969) models show that the magnetic field expansion up to the source surface plays a crucial role in determining global solar wind outflow properties (e.g., Wang 2009), including the terminal velocity, which is inversely correlated to the expansion factor itself. Wang and Sheeley (1990) concluded that the slow wind is produced from the edges of coronal holes. The insitu narrow boundary between fast and slow wind would therefore not be directly associated with a magnetic boundary in the corona (Suess et al. 1998). The slow solar wind has, however, distinctive compositional properties (Geiss et al. 1995; von Steiger et al. 2000; Ko et al. 
2006) that argue for a different origin of this plasma outside coronal holes. One source may be the large-scale current and plasma sheet associated with helmet streamer cusps-where plasmoid ejections have been observed (Sheeley et al. 1997; Jones and Davila 2009). The filling factor of the slow wind in the heliosphere seems to be too large to arise only from the helmet streamer cusps (McComas et al. 2007). Therefore magnetic field, plasma transport, and instabilities involving processes at coronal hole boundaries and on the quiet Sun must be involved (Rappazzo et al. 2005; Bettarini et al. 2006). Coronal holes appear to rotate as a rigid body, which requires continual footpoint interchanges between open and closed field lines. Other slow wind sources might be small, short-lived open regions interacting with the closed corona (e.g., Zurbuchen 2007), transient coronal holes neighboring active regions (Neugebauer and Liewer 2003), or more complex transport associated with the fractal meandering of coronal hole boundaries. Antiochos et al. (2012) identified the source of the slow wind at the Sun as a network of narrow (possibly singular) open-field corridors that map to a web of separatrices and quasi-separatrix layers in the heliosphere.

SPP will provide comprehensive in-situ measurements of solar wind plasma and coronal imaging that, combined with tomographic reconstruction of the global and fine-scale coronal structure and modeling, will help us to determine the connectivity of the fast, slow, and transient solar winds to their respective solar source regions. Measurements of plasma distributions and composition and of electric and magnetic fields will help identify signatures of the physical processes involved in wind formation, determine the magnetic connectivity to the source regions and establish the type of source region(s). Plasma and magnetic field data acquired by SPP during its multiple crossings of the region where the heliospheric equatorial current sheet develops close to the Sun will be used to directly measure breaks in the solar connectivity inside plasmoids originating at helmet streamers.

\subsubsection{Are the Sources of the Solar Wind Steady or Intermittent?}

Available solar wind measurements indicate a continuous outflow, punctuated by transients such as interplanetary coronal mass ejections (ICMEs) and shocks. There is significant evidence, however, for an intrinsic intermittency in solar wind generation. The slow solar wind at $1 \mathrm{AU}$ is bursty and variable over periods from days down to hours (Padhye et al. 2001; Burlaga et al. 2003). Interchange reconnection models for solar wind generation explicitly assume an intrinsic intermittency at the solar wind source in the form of reconnection between open field lines and closed coronal loops (e.g., Fisk 1996; Schwadron and McComas 2003; Suess et al. 1998; Antiochos et al. 2010). Solar eruptive events, which are known to contribute to the bursty solar wind, form a continuous spectrum in size (Robbrecht et al. 2009), and the fractionation that gives rise to measurable compositional signatures is likely size dependent. SPP direct in-situ measurements and local imaging of the coronaheliosphere boundary will identify the nature of the distribution and the importance of small ejecta that cannot be resolved at 1 AU. Even the fast solar wind may have a bursty origin in small-scale reconnection events at chromospheric height. This has been suggested to explain the inverse correlation between wind speed and both electron temperature (Gloeckler et al. 2003) and compositional enhancement of low-FIP (First Ionization Potential) elements (Zurbuchen et al. 2002). The high-speed solar wind is also intermittent (Feldman et al. 1997) and includes microstreams and pressure balance structures that are seen via radio scintillation (Grall et al. 1996) and in in-situ observations (Neugebauer et al. 1995). The variation may be due to instabilities that cause turbulence at high solar altitudes (e.g., Parhi et al. 1999) or to intermittent injection mechanisms. To date it has not been possible to determine the origin and variability of the fast solar wind as connections between solar events and high-speed wind features have not been adequately measured. 


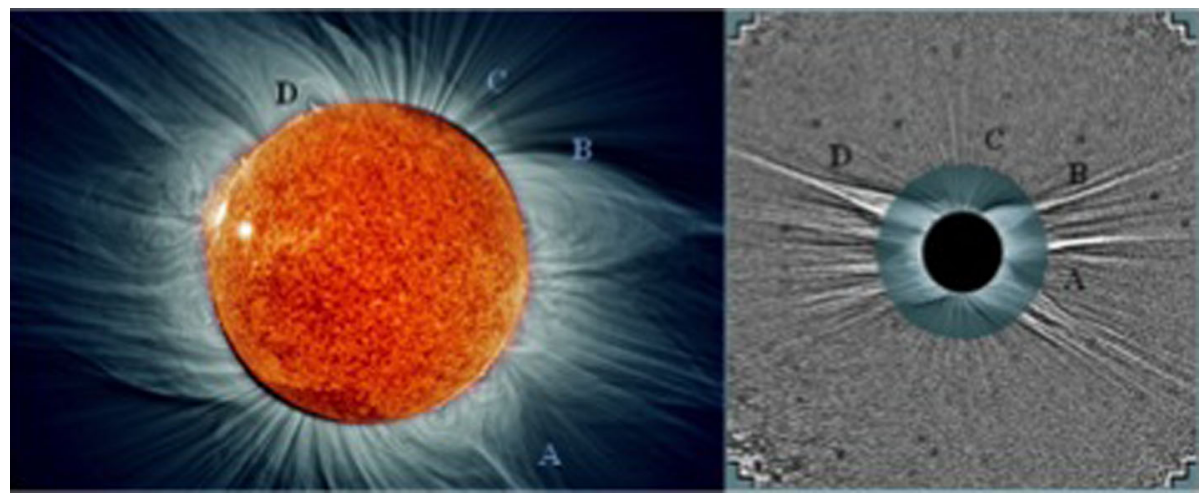

Fig. 7 SPP will provide the first direct in-situ evidence of how these structures merge into the solar wind in the heliosphere. Fine-scale structure in the solar corona: (A) Pseudo-streamers; (B) Helmet streamers; (C) Polar Plumes; (D) Prominence and prominence cavity below a streamer. Adapted from Wang et al. (2007)

During crossing of high-speed solar wind streams near perihelia, SPP should detect insitu remnants of jets, measuring durations and filling factors. Analysis of plasma and magnetic field data will identify signatures of interchange reconnection of closed field lines, at various heights, with dominant polarity open field lines, in the form of reconnection exhausts (Gosling 2007) and kinked field lines. Kinked field lines have been measured in the fast wind at Ulysses (Balogh et al. 1999), but an alternative interpretation to coronal reconnection as the stretching of field lines by shears in the wind, for example, on coronal plumes, has been suggested (Landi et al. 2006). If interchange reconnection is a ubiquitous process in the nascent solar wind, the number and duration of measured field line kinks should increase dramatically with proximity to the Sun. During the "fast radial scan" portion of SPP orbits temporal signatures of solar wind injection on multiple scales should be readily visible. Each orbit will yield two sets of in-situ measurements (one inbound and one outbound) of the variation of solar wind composition and density, electric and magnetic fields, and electron temperature along a particular heliospheric flow tube, sampling all major wind types over the course of the mission.

\subsubsection{How Do the Observed Structures in the Corona Evolve into the Solar Wind?}

Off-limb observations show the presence of ubiquitous ray-like structures emanating not only from active regions (i.e., streamers and pseudo-streamers) but also from coronal holes (i.e., coronal plumes as illustrated in Fig. 7). Although these structures can be traced to several solar radii above the solar surface, it remains unclear how they evolve into the solar wind. Their respective contributions to the solar wind has proven to be hard to quantify from distant (e.g., $1 \mathrm{AU}$ ) observations. For instance, the structure and dynamics of coronal plumes are not well understood, though they appear to be correlated with small-scale bipolar structures embedded in the overall unipolar coronal hole magnetic field (Wang and Sheeley 1995; Raouafi and Stenborg 2014). DeForest et al. (2001) showed that coronal plumes are episodic in nature and recur for days and maybe weeks above the same regions in coronal holes. Raouafi et al. (2008) used STEREO/EUVI and Hinode/XRT observations to show that coronal jets are precursors of coronal plume formation and in a recent work Raouafi and Stenborg (2014) showed that plume footpoints are dominated by high-frequency, small-scale jet-like activity (i.e., jetlets). Ultraviolet measurements seem to show they are cooler than 
the surrounding background hole plasma — close to their base, coronal plumes have electron temperatures of about $1 \mathrm{MK}$, slightly increasing with height, while inter-plume lanes are at 2 MK or more - and have slower, but denser outflows (e.g., Wilhelm 2006; Raouafi et al. 2007). Plumes can be traced up to several tens of solar radii (i.e., $\sim 30 R_{S}$; Deforest et al. 1997). Their contribution to the solar wind remain, however, controversial.

The correspondence between coronal and 1 AU solar wind fine-scale structures is masked by the dynamic evolution of the wind. Using magnetic field, plasma, and composition measurements closer to the Sun, SPP will link magnetic field variations as well as those in the solar wind plasma directly to remotely observed coronal structures. In addition, during the time intervals when SPP will nearly corotate with the Sun when moving radially towards or away from the Sun, the timescales of longitudinal and radial structures will be decoupled, making it possible to distinguish between temporal variability and spatial structure. SPP plasma and magnetic field measurements will enable us to identify the coronal sources of fast and slow wind over an extended period and to determine how current sheets and other wind structures such as magnetic holes and plasma sheets form.

\subsection{Explore Mechanisms that Accelerate and Transport Energetic Particles}

The acceleration and transport of solar energetic particles (SEPs) has been studied extensively (e.g., Reames 1999; Mewaldt 2006; Lee et al. 2012; Raymond et al. 2012; Drake and Swisdak 2012; Giacalone 2010). Discriminating among possible acceleration processes at and near the Sun using data acquired only at 1 AU has proven difficult. Particle propagation through the solar wind en route to $1 \mathrm{AU}$ distorts initially time-ordered structures and energy-spectral features produced by putative particle acceleration processes and mixes particles from different acceleration sites. SPP will help determine the origins of SEPs and the operative transport processes, identify the seed particle populations available for further acceleration to high energies both near the Sun and in the solar wind, and distinguish among candidate processes of particle acceleration on the Sun and in the heliosphere. SPP will measure the intensities, energy spectra, and pitch-angle distributions of energetic electrons, protons, and heavy ions in the innermost region of the heliosphere (McComas et al. 2014). Particle distributions measured in-ecliptic in the helioradius range 9.86-53.7 $R_{S}$, where SPP will perform its prime science measurements, are expected to show less distortion caused by propagation effects than distributions measured further from the Sun and therefore help us to identify the acceleration mechanisms.

\subsubsection{What Are the Roles of Shocks, Reconnection, Waves, and Turbulence in the Acceleration of Energetic Particles?}

Figure 8 depicts the current paradigm for the origin of SEP events. Energization is thought to occur mainly in solar flares, e.g. by resonant acceleration in cascading turbulent fluctuations from magnetic reconnection (Raymond et al. 2012), and by diffusive shock acceleration at CME-driven shock waves (Lee 2005). The ambient solar wind contains relatively low intensities of low-frequency waves that are gyro-resonant with SEPs. But in the sheath adjacent to and upstream of a shock, particles streaming along $\mathbf{B}$ can excite high intensities of gyro-resonant waves, which in turn scatter the particles in pitch angle and produce the small scattering mean free paths essential for shock acceleration (Lee 2005). Figure 9 shows an example of such waves measured upstream of a quasi-parallel shock (angle $\theta_{B_{n}}$ between the shock normal and $\mathbf{B}$ satisfies $\theta_{B_{n}} \leq 45^{\circ}$; Tsurutani et al. 1983, see also Kennel et al. 1984). 


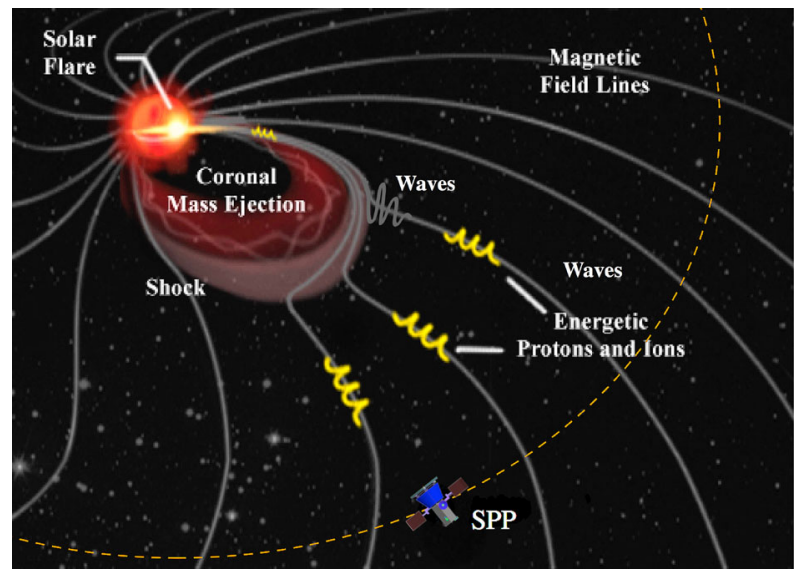

Fig. 8 SPP, shown along its orbit (dashed curve) near a perihelion pass, will measure solar energetic ions and electrons from a vantage point very near the site where these particles are accelerated. The illustration sketches the occurrence of a solar flare and a CME extending a few $R_{S}$ from the Sun. The shock at the front edge of the CME and the compressed sheath plasma behind the shock form as the CME, with its entrained flux rope (tangled pink lines), pushes outward from the Sun through the ambient solar wind. Swept-up magnetic field lines are refracted and compressed across the shock and draped around the CME. Energetic particles accelerated at both the flare and CME shock are shown spiraling away from the Sun (yellow spirals) along the magnetic field. For simplicity, magnetic field lines around the shock are depicted as smooth. However, it is expected that the field ahead of CME shock and in the sheath will highly structured. Waves ahead of the shock that are produced by high intensities of shock-accelerated ions streaming away from the shock are sketched for the uppermost magnetic field line connected to the CME shock. Adapted from http://spaceweather. uma.es/solarstorms.html

The near radial orientation of the $\mathbf{B}$ relative to the upper portion of the shock front in Fig. 8 suggests a quasi-parallel shock near its nose with a precursor of magnetic fluctuations, as sketched in the figure. Shock-accelerated SEPs near the Sun can become intense enough to exert a back reaction on, and thereby self-consistently modify, the shock structure, and affect in turn the conditions for particle injection, acceleration, and escape at and near the shock. Other possible particle acceleration mechanisms include also (i) statistical Fermi process in which particles are resonantly scattered by Alfvén waves with wavelengths comparable to the particle gyroradius (Jokipii 1971), (ii) transit time damping in which particles interact with propagating fluctuations in the magnitude of the interplanetary magnetic field (e.g., magnetosonic waves that are less damped in a magnetized low-beta plasma where the sound speed is lower than the Alfvén speed) (Fisk 1976), (iii) stochastic acceleration as particles move through a magnetic medium undergoing random compressions or expansions (Fisk and Gloeckler 2006; Jokipii and Lee 2010; Antecki et al. 2013; Zhang and Lee 2013); and (iv) acceleration by gradual solar wind compression regions (Giacalone et al. 2002). Stochastic acceleration processes due to magnetohydrodynamic waves will likely be important close to the Sun, especially below the Alfvén point, where presumably wave amplitudes can be large and shocks may not form as quickly because of the low Mach number flows.

SPP will measure CME shocks where particle acceleration to high energies (protons to $10 \mathrm{GeV}$, electrons to a few tens of $\mathrm{MeV})$ is thought to maximize $\left(R<20 R_{S}\right.$; Kahler 1994; Mewaldt et al. 2012). Measurements made near SEP acceleration sites will reduce uncertainties due to modifications of angular distributions by propagation. This will provide the timing needed to differentiate specific acceleration processes and parti- 

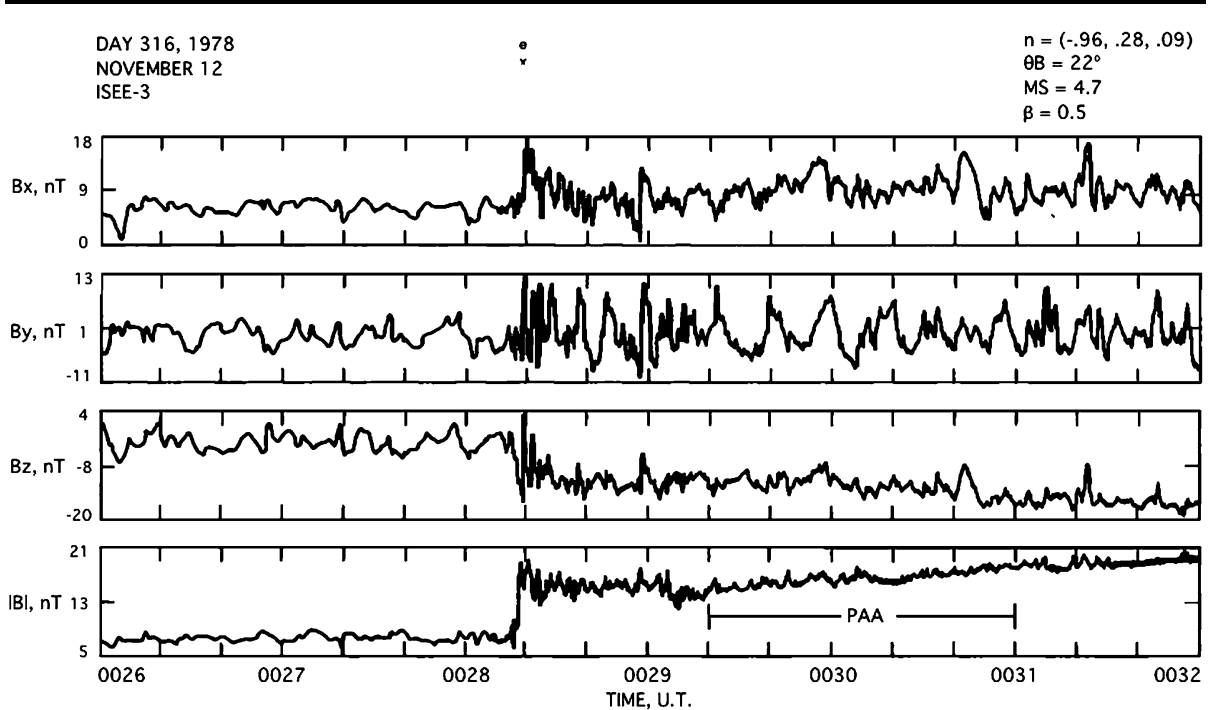

Fig. 9 SPP will provide quantitative determination of the role of "coronal" shocks in the injection and acceleration of solar energetic particles, and will measure the power spectral densities of the hydromagnetic waves that are generated in a self-consistent way by the particles. These waves, in turn, scatter the particles, causing the particles to cross the shock multiple times and undergo large energy increases. This figure shows magnetic field observations of a quasi-parallel $\left(\theta_{B_{n}}=22^{\circ}\right)$ interplanetary shock observed at 1 AU by ISEE-3. In the bottom panel the shock passage at $\approx 00: 28: 06$ UT is accompanied by an increase in the magnetic field magnitude $|\mathbf{B}|$. The Cartesian components $\left(B_{x}, B_{y}, B_{z}\right)$ of the field (top three panels) show large-amplitude fluctuations ahead of the shock generated by energetic ions streaming away from the shock along the mean magnetic field (Kennel et al. 1984). From Tsurutani et al. (1983)

cle sources. An important task for SPP is to determine to what extent SEP acceleration in the low corona is mediated by the energetic particles. Measurements of particle mass, energy, and pitch-angle taken at high-time resolution together with those of the plasma and magnetic field at comparable time resolution are required for a precise determination of particle injection and acceleration processes at the shocks and the possible mediation of the shock by the particles themselves. SPP will also measure lower intensity SEP events that are produced by small impulsive solar flares and usually go undetected at $1 \mathrm{AU}$ because of their low SEP intensities. The continuous versus bursty character of these events when measured during different levels of solar activity may provide evidence of the processes that fill the heliosphere with low energy particles, even during the quietest periods.

\subsubsection{What are the Source Populations and Physical Conditions Necessary for Energetic Particle Acceleration?}

Observations at 1 AU show a continual outflow of ions with energies extending from $>1 \mathrm{keV} \mathrm{nuc}^{-1}$ to $\sim 10 \mathrm{MeV}$ nuc $^{-1}$ (Gloeckler 2010). These ions may constitute an important contribution to the seed population for SEPs. Suprathermal ions in the solar wind may indicate a quasi-continuous ion acceleration process at the Sun or in the solar wind, or both. Figure 10 shows the differential intensity versus energy per nucleon of protons (circles) and oxygen ions (triangles) measured at $1 \mathrm{AU}$ under different solar conditions 


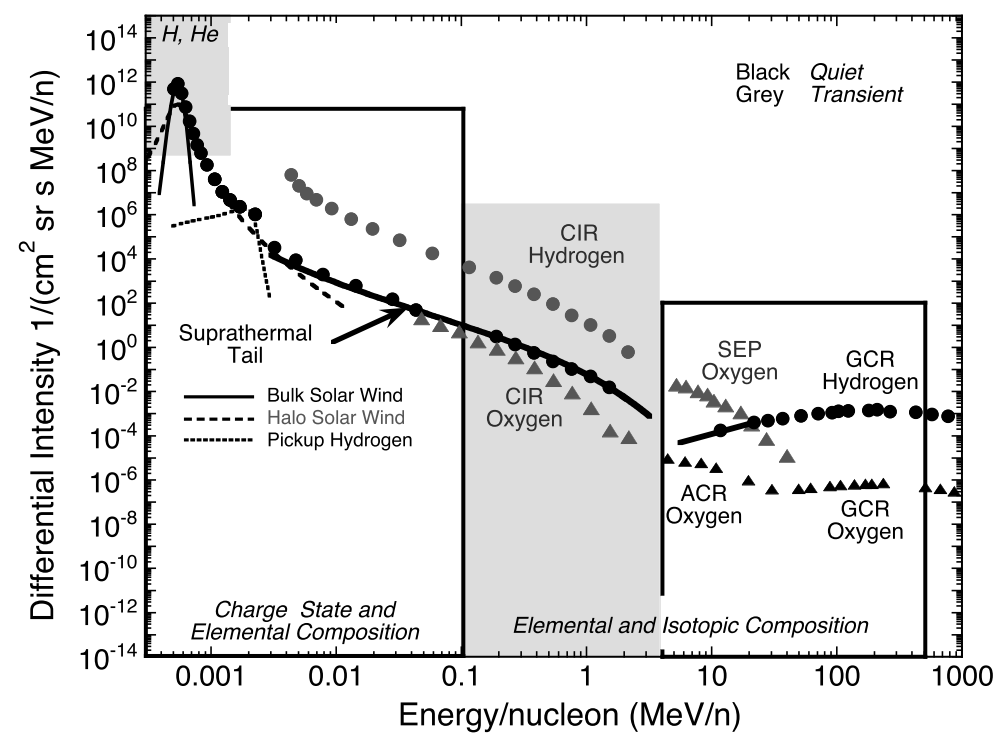

Fig. 10 SPP will provide continuous measurements of suprathermal populations and solar wind parameters, which will allow us to characterize (i) the intensity and composition of suprathermal seed particles, (ii) the physical conditions necessary for particle acceleration, and (iii) the relative contributions of remnant impulsive flare material, direct flare material, solar wind and suprathermal material, or CME material into the shock acceleration processes. The figure shows energetic particle measurements from the ACE spacecraft at $1 \mathrm{AU}$ integrated over quiet (black) and active (grey) periods. Intensities represented are of protons (circles) from solar wind energies to those of galactic cosmic rays (GCRs), and of oxygen ions (triangles). From Gloeckler (2010)

(Gloeckler 2010). This figure emphasizes the broad range of energies and very large dynamic range of observed ion intensities. Moreover, close to the Sun it would be possible to directly observe the inner source of pickup ions close to their origin (Schwadron et al. 2000). Comparison of SEP compositions with those of both suprathermal and solar wind ions will enable us to quantify the relative contributions of remnant impulsive flare material, direct flare material, solar wind and suprathermal material, or CME material into the shock acceleration process. The continuous measurements of suprathermal populations and solar wind parameters will help us determine the conditions under which suprathermal tails form and therefore test the mechanisms proposed for their formation (Fisk and Gloeckler 2012; Zhang and Lee 2013; Antecki et al. 2013; Lynn et al. 2013; Randol and Christian 2014; Jokipii and Lee 2010; Tessein et al. 2013, and references therein).

With its multiple orbits, SPP will allow us to estimate the contribution of the acceleration processes described above in the development of the SEP events and in building the suprathermal tails in regions never before visited, and do so over a large fraction of the solar cycle under a wide range solar wind flow conditions (McComas et al. 2013, 2014). SPP measurements of low-energy solar electrons and ions during different levels of solar activity will increase our understanding of where and how the seed particles in suprathermal tails of VDFs are energized. Near its closest perihelia passes, SPP will be much closer to the energetic particle acceleration sites at the Sun than are current observation platforms at $1 \mathrm{AU}$. This proximity will reduce the uncertainties in the estimated timing of particle release on the Sun. 


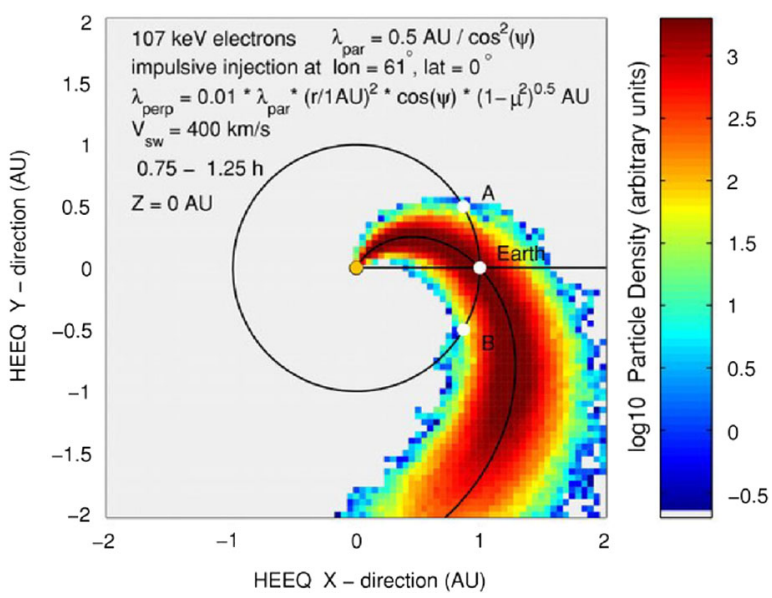

Fig. 11 SPP measurements of SEP energy spectra and the ambient and particle-generated electromagnetic turbulence over a large fraction of the solar cycle will determine how scattering properties from the low corona into the solar wind vary with magnetic field intensities, turbulence levels, and solar wind parameters and specify what role transport processes play in the distribution of SEPs. The figure shows simulated distribution of $107 \mathrm{keV}$ electrons in the equatorial plane $\sim 4 \mathrm{~h}$ after impulsive injection from solar latitude $0^{\circ}$, solar longitude $61^{\circ}$, and helioradius $0.05 \mathrm{AU}$, using a constant ratio of the parallel to perpendicular scattering mean free paths, and simulation parameters given in the legend. From Dröge et al. (2010)

\subsubsection{How Are Energetic Particles Transported in the Corona and Heliosphere?}

Observations of ${ }^{3} \mathrm{He}$-rich impulsive events from spacecraft widely separated in azimuth (Wiedenbeck et al. 2013) suggest that cross-field transport in the corona and/or solar wind may be responsible for distributing SEPs injected from well-localized sources. Alternatively, special magnetic connectivity of the spacecraft to a particle source of a given angular extent is required to explain the wide spread of SEPs. For example, Dröge et al. (2010) investigated the combined effects of SEP propagation parallel and perpendicular to the large-scale solar wind magnetic field by solving numerically the focused-diffusion transport equation (e.g., Isenberg 1987). Figure 11 shows the azimuthal and radial intensity that Dröge et al. (2010) predicted for $107 \mathrm{keV}$ electrons in the ecliptic plane. Good pitch-angle coverage during the observation of these small events close to the Sun will allow SPP to determine whether the longitudinal spreading of these SEP events is due to a direct magnetic connection to the particle source or because of other transport mechanism(s) (e.g., cross-field diffusion). In addition, the synergy of SEP measurements from SPP and 1 AU spacecraft will help constrain the role of cross-field transport within 1 AU.

SPP will measure the evolution of SEP energy spectra and angular distributions at helioradii from $<10 R_{S}$ to beyond $\sim 0.25 \mathrm{AU}$. These data in combination with measured ambient and particle-generated electromagnetic turbulence will determine how scattering properties from the low corona into the solar wind vary with magnetic field intensities, turbulence levels, and solar wind parameters over a large fraction of the solar cycle. SPP will search for near-Sun evidence of the roles played by particle diffusion and wave amplification in producing SEP events, not only very near CME-driven shocks where proton-amplified Alfvén waves can trap particles undergoing acceleration, but also further from acceleration sites where escaping particles carry spectral, compositional, and angular signatures of the acceleration and propagation processes. 


\section{SPP Observations, Investigations and Requirements}

In this section we will discuss the science in-situ and remote sensing measurements to be returned by the SPP mission. We also present a brief description of the instruments and the requirements. The detailed specifics of the instruments are covered in the accompanying instrument papers (McComas et al. 2014; Vourlidas et al. 2015; Bale et al. 2015, this issue; Kasper et al. 2015, this issue; hereafter M2014, V2015, B2015, and K2015, respectively).

\subsection{Science Observations}

The SPP mission will measure the fluctuations and variations of the magnetic and electric fields in the inner heliosphere to within $10 R_{S}$. The measurements cadence and sensitivity will be sufficient to identify and characterize solar wind structures (e.g., shocks, current sheets, discontinuities, and CMEs); to analyze fluctuations and turbulence down to the dissipation range; and to identify and quantify the energy flux in the waves that are responsible, at least partially, for the heating and acceleration of the coronal and solar wind plasma. SPP will derive the properties of the thermal solar wind electron, proton and alpha particles (e.g., density, velocity, and temperature). The energy range of observations will distinguish between slow and fast solar wind streams and resolve CMEs. Remote-sensing imaging from SPP will map and resolve with sufficient spatial resolution and cadence the morphology, velocity, acceleration, and mass density of evolving solar wind structures. These images will provide context for the in-situ measurements and will help understanding of the connection of the solar corona to the inner heliosphere. The measurement products will enable the scientific goals of inferring the heating mechanisms of the corona and the solar wind; determining the structure of the inner heliosphere including narrow jets and streams as well as the sources of the solar wind; and quantifying the effect of the variable fields on energetic particle acceleration and transport.

The SPP mission will determine the intensity, spectrum and pitch-angle distribution of energetic electrons, protons and heavy ions in the innermost part of the heliosphere where propagation effects are minimized and particle acceleration processes are still in progress. Energetic particles will be measured with a sufficient cadence and sensitivity to identify various processes of particle acceleration, particle distributions through the corona, and source populations on the Sun and in interplanetary space. These products will enable the scientific goals of understanding and quantifying the physical conditions necessary for particle energization, acceleration, and transport from the corona throughout interplanetary space.

\subsection{Science Requirements}

This section describes the baseline requirements for the SPP mission. To meet the science objectives, the SPP mission will spend at least 920 hours below $20 R_{S}$ heliocentric, with no less than 14 hours below $10 R_{S}$. There are no specific instrument requirements at the highest level but rather everything is written in terms of measurement requirements to allow maximum mission robustness. The required measurements are detailed in the accompanying instrument papers (M2014; K2015; V2015; and B2015). The measurements will be made at least between heliocentric minimum perihelion of $<10 R_{S}$ and $0.25 \mathrm{AU}$. One key to the success of SPP is the in-situ sampling of the region inside of roughly $20 R_{S}$ of subAlfvénic solar wind (Fig. 12) where the coronal magnetic structure still channels the flow and determines angular momentum loss, and where waves and turbulence are strongest. This is also the region where transitions from collisional to collisionless plasma and from magnetic to kinetic pressure occur. 


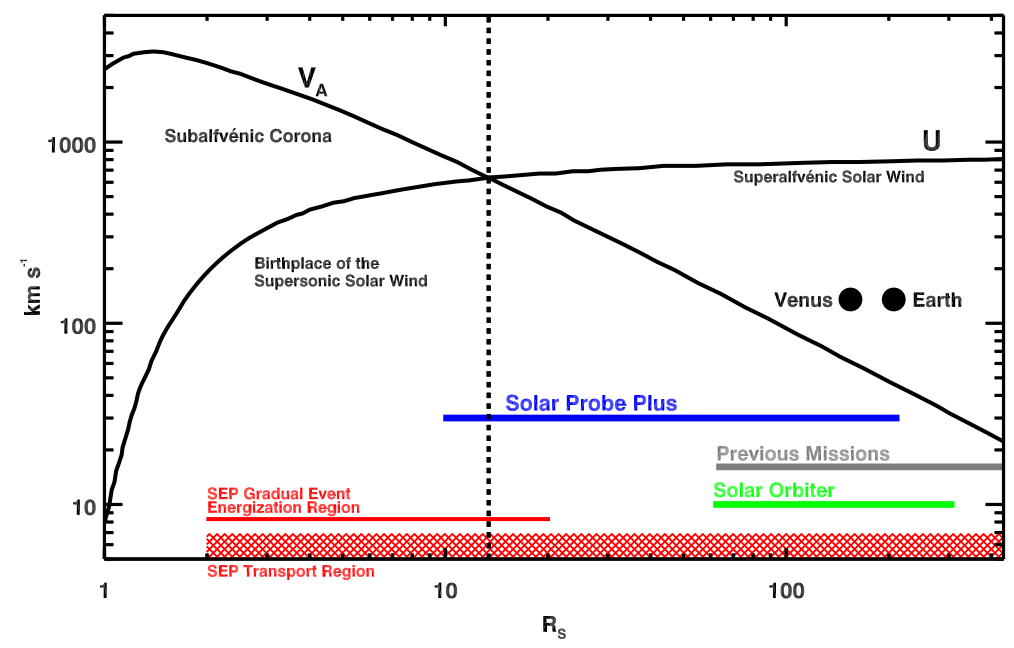

Fig. 12 SPP will be the first mission to fly inside the solar wind source region and to sample directly the critical region of the outer corona where solar energetic particles (SEPs) are generated. The Solar Orbiter mission will also observe the Sun and the solar wind several 10s of degrees above the ecliptic and will provide complementary measurements to those of SPP. Model profiles of solar wind speed U and Alfvén wave speed $\mathrm{V}_{\mathrm{A}}$ versus helioradius. The vertical bar separates the source, or sub-Alfvénic, region of the wind from the super-Alfvénic solar wind flow

The required measurements include magnetic and electric fields, plasma waves, quasithermal noise and radio emissions, thermal ions and electrons, energetic electrons, protons and heavy ions, and visible broadband images. The required observations are such that the 3 science objectives of the SPP mission and all of their underlying science questions (Sect. 2) can be fully addressed. However, it is recognized that with the inclusion of models we can still make sizable progress in answering these key questions with a reduced set of measurements. The science can be addressed with different combinations of measurements and, furthermore, there are multiple schemes of obtaining the measurements using different instrument combinations. Thus, the mission is robust against the failure of any instrument or the loss of any given measurement after launch.

Table 1 illustrates the different types of measurements that can be combined in multiple ways to address each of the SPP science objective questions. These combinations present sufficient flexibility to provide outstanding science return even for the unlikely case of degradation of the spacecraft and/or instruments, and provide multiple paths to successfully address the SPP science questions. The instruments used to make the various measurement types are described in the next section.

\subsection{Science Investigations}

The SPP instrument Science Investigations, selected by NASA in September 2010, are: the Electromagnetic Fields Investigation (FIELDS); the Integrated Science Investigation of the Sun, Energetic Particle Instruments (ISIS); the Solar Wind Electrons Alphas and Protons Investigation (SWEAP); and the Wide Field Imager for Solar Probe Plus (WISPR). The institutions participating with the instrument science investigations are listed in Table 2. In addition to the four instrument investigations, there is also a theory and modeling investigation-Heliospheric origins with Solar Probe Plus (HeliOSPP). The HeliOSPP PI, 


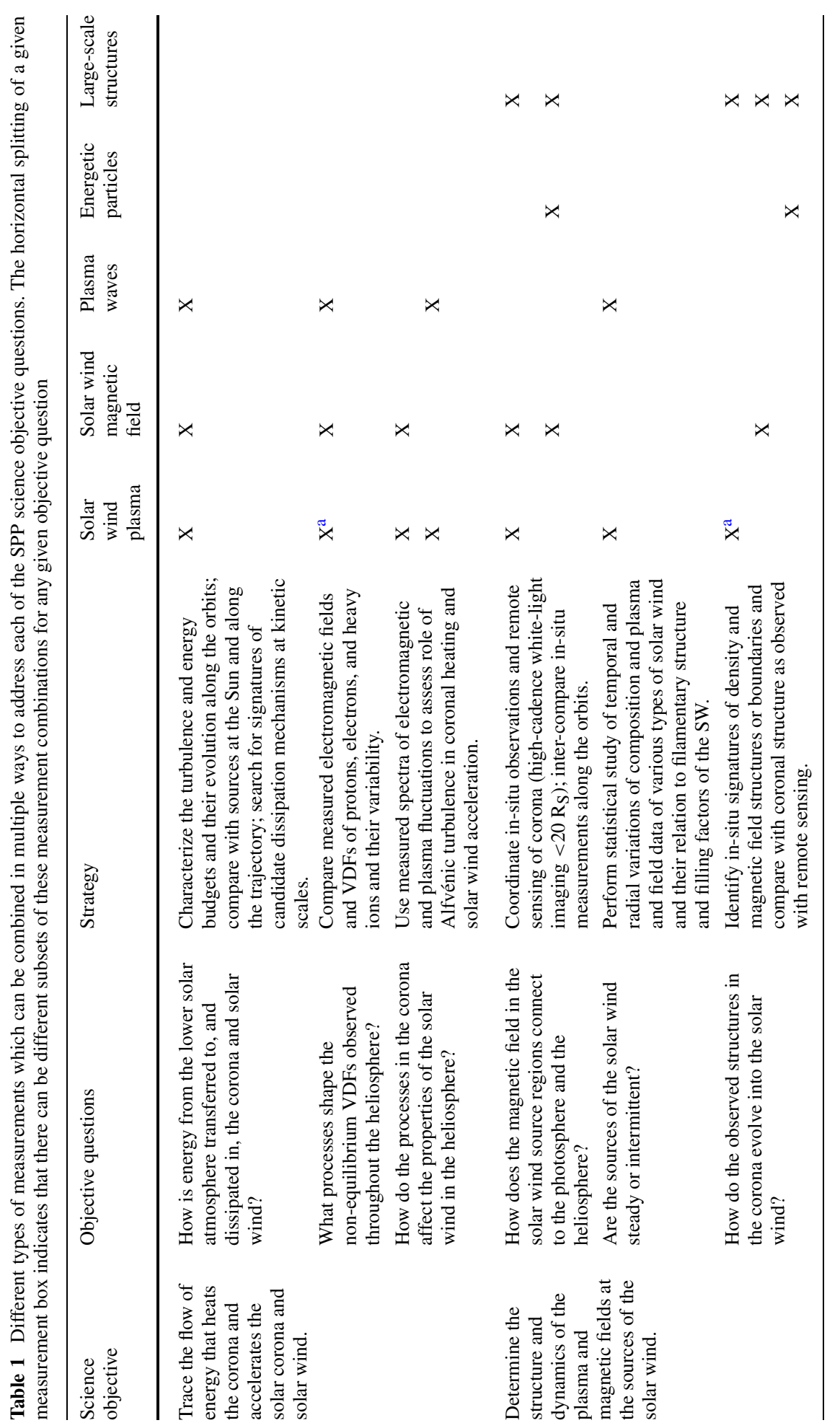




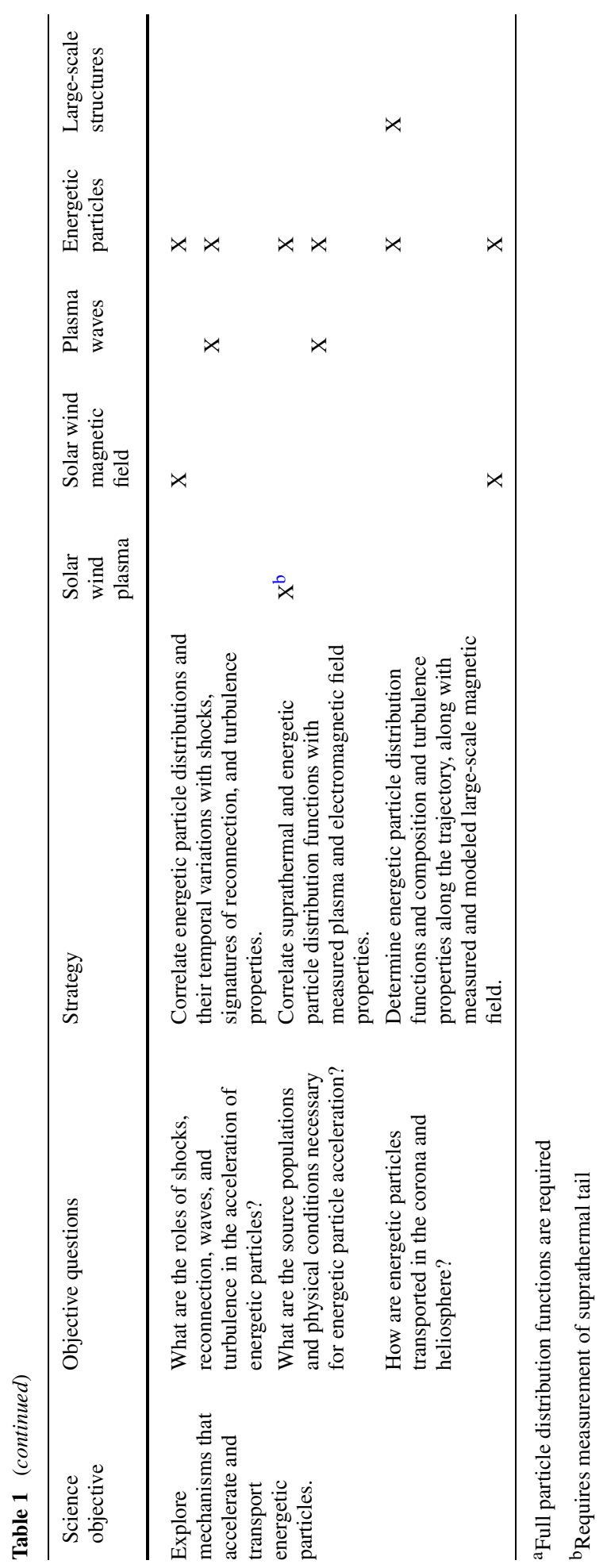


Table 2 SPP science investigations, lead institutions, and team institutions

\begin{tabular}{lll}
\hline Science investigations & Lead institutions & Team institutions \\
\hline FIELDS & $\begin{array}{l}\text { Univ. California, } \\
\text { Berkeley }\end{array}$ & $\begin{array}{l}\text { CNRS/LPC2E, Orleans, France; NASA/GSFC; Obs. Meudon, } \\
\text { France; SAO; Univ. Colorado; Univ. Maryland, College Park; } \\
\text { Univ. Minnesota; Univ. New Hampshire }\end{array}$ \\
& SwRI & Caltech, JPL; JHU/APL; NASA/GSFC; Univ. Delaware; Univ. \\
& New Hampshire; Univ. Arizona
\end{tabular}

Dr. Marco Velli, serves as the Observatory Scientist for the SPP Project, and carries out an inter-disciplinary science investigation that focuses on the goals and objectives of the SPP mission. He provides theoretical input and independent assessment of scientific performance to the Science Working Group (SWG) and the SPP Project to maximize the scientific return from the mission.

The FIELDS investigation (B2015) comprises two fluxgate magnetometers, a search coil magnetometer and five electric antennas measuring electric and magnetic fields and waves, spacecraft floating potential, density fluctuations, and radio emissions.

The SWEAP investigation (K2015) has two electrostatic analyzers and a Faraday cup. This investigation will count the most abundant particles in the solar wind-electrons, protons and helium ions - and measure their properties such as velocity, density, and temperature.

The ISIS energetic particle instrument suite is composed of two independent instruments (EPI-Hi and EPI-Lo) covering different (and overlapping) energy ranges (M2014). This suite will make observations of energetic electrons, protons and heavy ions that are accelerated to high energies (10 s of keV to $100 \mathrm{MeV}$ ) in the Sun's atmosphere and inner heliosphere.

The WISPR white light telescope will take images of the solar corona and inner heliosphere (V2015). The experiment will also provide images of the solar wind, shocks and other structures as they approach and pass the spacecraft. This investigation complements the other instruments on the spacecraft providing direct measurements by imaging the plasma the other instruments sample.

As noted above, the science requirements are written in terms of measurements allowing multiple ways of obtaining the measurements listed in Table 1 using different instrumentation. Multiple SPP instruments contribute to the measurements of vector magnetic fields, solar wind plasma, plasma waves and energetic particles.

The FIELDS fluxgate magnetometer provides measurements of the DC and low frequency fluctuating magnetic fields. The direction of the magnetic field can also be determined using electron pitch angle distributions from SWEAP (K2015) or ISIS/EPI-Lo (M2014), since electron pitch angle distributions tend to be organized by the local magnetic field direction, a technique that has been successfully applied to many prior spacecraft measurements (e.g., Rème et al. 1986; Lin et al. 1995). Moreover, the FIELDS electric field measurements (B2015) can also be used to determine the local vector magnetic field in the highly conducting solar wind plasma. 
The solar wind plasma conditions will be measured by the SWEAP Solar Probe ANalyzer (SPAN) top-hat electrostatic analyzer and the Solar Probe Cup (SPC) Faraday cup (K2015). The FIELDS plasma wave instrument, identifying the local electron plasma frequency, will also independently and very accurately determine the solar wind plasma density. ISIS/EPILo angular distributions will provide estimates of solar wind flow velocity (M2014). Moreover, the WISPR white light imager (V2015), when close to the Sun $\left(<55 R_{S}\right)$, will also provide a measure of the line-of-sight integrated solar wind plasma density and velocity.

Plasma wave measurements of electric and magnetic fields are provided by the FIELDS instrument suite composed of the electric field antennas, the search coil and the fluxgate magnetometer (B2015). The solar wind fluctuations will also be measured by the SWEAP instrument suite (K2015) and imaged by WISPR (V2015).

The science questions call for a wide range of energetic particle energy measurements. The ISIS suite (M2014) has two energetic particle instruments, which provide wide-energy coverage, and the overlap between the two, together with the overlap with the SWEAP instruments provides multiple ways to measure parts of the required spectrum.

The WISPR instrument will provide white light images showing the large-scale structures in the solar wind (V2015). The FIELDS measurements of the turbulent flows can also be used to provide information on the size and type of structures being sensed by the SPP mission (B2015).

Thus, while all of the SPP instruments are required to accomplish full mission success and specifically the baseline science objectives, the mission is robust against the unlikely loss of any instrument and minimum mission success will still be reached.

\section{Mission Design}

To accomplish the science objectives, the SPP spacecraft will fly through a region of space near the Sun that offers difficult and unique challenges. First, getting near the Sun requires a high-energy launch and insertion into an orbit designed to allow for significant periods of time in the region of interest; the mission design also constrains spacecraft resources such as mass and power. Second, the physical environment near the Sun and the unforgiving nature of the outer corona drive the spacecraft design, leading to significant technology development, autonomous operations during solar encounter, and unique engineering challenges. Finally, the orbital dynamics of SPP and Earth impose a geometry on mission operations and communication that further drive the autonomous operations to collect science data, and complex uplink/downlink plans to retrieve that data. We discuss each of these aspects of the mission below.

To reach regions of space below $10 R_{S}$ from Sun center, SPP uses a Venus intercept launch trajectory, allowing a gravity assist about 6 weeks after launch for insertion into an elliptical orbit around the Sun with perihelion near $35 R_{S}(0.16 \mathrm{AU})$ and aphelion near 1 AU. Six subsequent Venus gravity assists through a total of 24 orbits decrease the perihelion in stages to a final perihelion at $9.86 R_{S}(0.0459 \mathrm{AU})$ in the final three orbits. Launching between 31 July and 18 August 2018, the trajectory is designed to complete the mission science objectives over 7 years. Figure 13 shows the trajectory and timeline for initial and final perihelia and all seven Venus flybys. Figure 14 gives Sun-probe distance through the mission, focusing on the periods when the spacecraft is inside $53.7 R_{S}(0.25 \mathrm{AU})$ showing the effect of each Venus gravity assist on the following perihelia.

One of the benefits of the mission design is that each orbit is similar. The mission design takes advantage of this by dividing operations within an orbit into two phases-a solar 
Fig. 13 SPP trajectory viewed from above the ecliptic plane with launch date on July 31 , 2018. The seven Venus gravity assists (i.e., Venus Flybys [VF]; green dots) with the corresponding dates are also shown as well as first perihelion and the first minimum perihelion

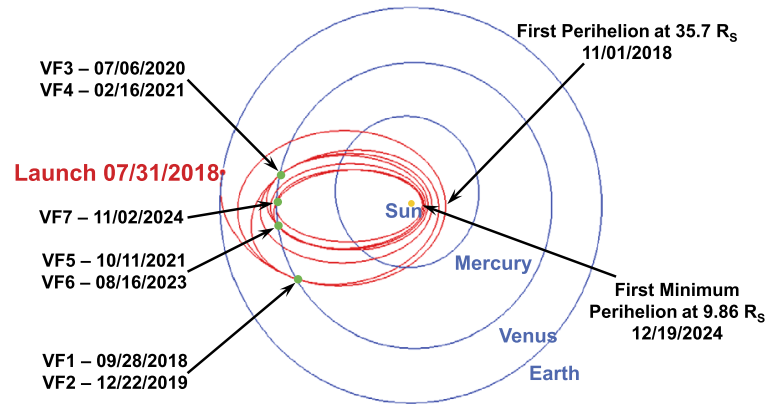

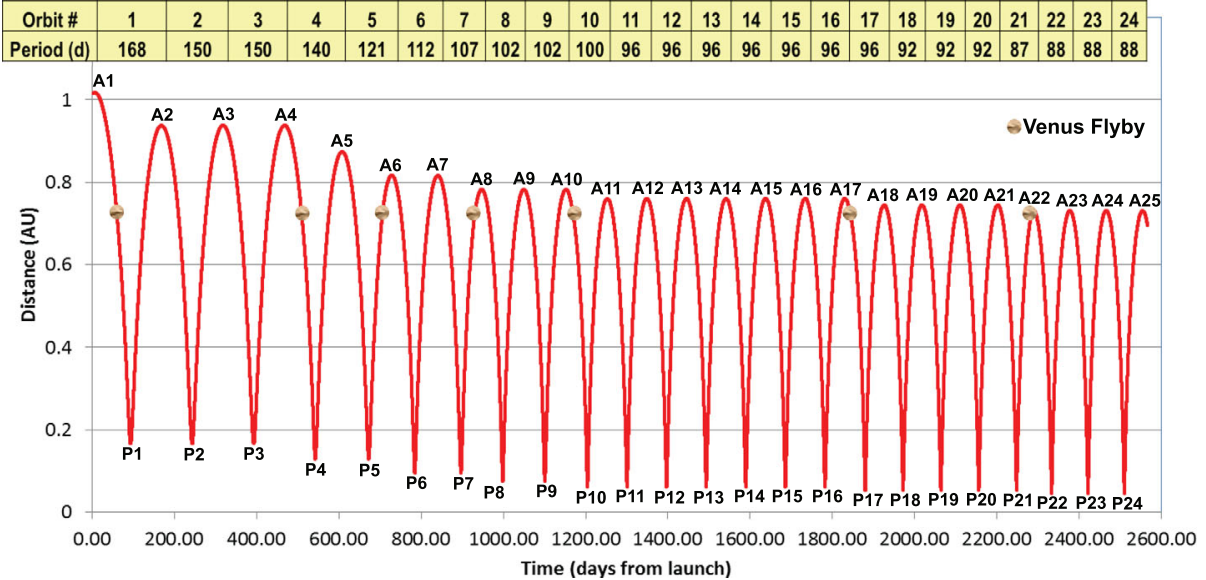

Fig. 14 SPP-Sun distance through the mission, showing walkdown to minimum perihelion. Venus flybys are indicated by the circular brown icons

encounter phase, that part of the orbit where the spacecraft is closer than $0.25 \mathrm{AU}$, and a downlink/cruise phase outside the solar encounter region. The bulk of the science operations take place during the solar encounter; during this time, instruments collect data and store measurements of interest on the spacecraft and instrument suite solid-state recorders. Communications are limited during these close approaches to the Sun, so science collection is autonomous and only limited health and safety telemetry are downlinked. Table 3 gives a breakdown of the time spent in various regions near the Sun. Outside the solar encounter period, the downlink/cruise phase is where all other activities needed to operate the mission take place, including science and housekeeping data downlinks, navigation contacts, trajectory correction maneuvers, and command uploads for autonomous activities. SPP will make science measurements during the downlink/cruise phase as power is available and when other activities are not in progress.

The SPP trajectory requires high launch energy, with maximum C3 over the launch period of $154 \mathrm{~km}^{2} \mathrm{~s}^{-2}$. To meet these requirements, SPP will launch on a Delta IV Heavy class launch vehicle and an upper stage based on the STAR-48B solid rocket motor. Launch will take place from Kennedy Space Center/Cape Canaveral Air Force Station. A backup launch period is planned in May 2019, in the unlikely event that the mission cannot launch during the primary period. If the backup launch is used, the trajectory of the mission will require an additional Venus gravity assist and two phasing orbits to insert SPP into an orbit similar 
Table 3 Orbit time spent below several radial thresholds within the Solar Encounter phase

\begin{tabular}{|c|c|c|c|c|c|c|}
\hline \multirow[b]{2}{*}{$\begin{array}{c}\text { Solar } \\
\text { Pass \# }\end{array}$} & \multirow[b]{2}{*}{$\begin{array}{l}\text { Perihelion } \\
\text { (AU) }\end{array}$} & \multirow[b]{2}{*}{$\begin{array}{l}\text { Perihelion } \\
\quad\left(\mathbf{R}_{\mathrm{S}}\right)\end{array}$} & \multicolumn{4}{|c|}{ Time within } \\
\hline & & & $\begin{array}{c}30 \mathbf{R}_{\mathrm{S}} \\
(\mathrm{hr})\end{array}$ & $\begin{array}{c}20 \mathrm{R}_{\mathrm{S}} \\
(\mathrm{hr})\end{array}$ & $\begin{array}{c}15 \mathrm{R}_{\mathrm{S}} \\
(\mathrm{hr})\end{array}$ & $\begin{array}{c}10 \mathrm{R}_{\mathrm{S}} \\
(\mathrm{hr})\end{array}$ \\
\hline 1 & 0.163 & 35.66 & & & & \\
\hline 2 & 0.163 & 35.66 & & & & \\
\hline 3 & 0.163 & 35.66 & & & & \\
\hline 4 & 0.128 & 27.85 & 61.04 & & & \\
\hline 5 & 0.128 & 27.85 & 61.04 & & & \\
\hline 6 & 0.092 & 20.35 & 104.22 & & & \\
\hline 7 & 0.092 & 20.35 & 104.22 & & & \\
\hline 8 & 0.072 & 15.98 & 108.55 & 48.06 & & \\
\hline 9 & 0.072 & 15.98 & 108.55 & 48.06 & & \\
\hline 10 & 0.06 & 13.28 & 107.27 & 55.13 & 24.75 & \\
\hline 11 & 0.06 & 13.28 & 107.27 & 55.13 & 24.75 & \\
\hline 12 & 0.06 & 13.28 & 107.27 & 55.12 & 24.75 & \\
\hline 13 & 0.06 & 13.28 & 107.27 & 55.13 & 24.75 & \\
\hline 14 & 0.06 & 13.28 & 107.27 & 55.12 & 24.74 & \\
\hline 15 & 0.06 & 13.28 & 107.27 & 55.12 & 24.74 & \\
\hline 16 & 0.06 & 13.28 & 107.27 & 55.12 & 24.73 & \\
\hline 17 & 0.052 & 11.42 & 105.03 & 56.91 & 32.23 & \\
\hline 18 & 0.052 & 11.42 & 105.03 & 56.91 & 32.23 & \\
\hline 19 & 0.052 & 11.42 & 105.03 & 56.91 & 32.23 & \\
\hline 20 & 0.052 & 11.42 & 105.03 & 56.91 & 32.23 & \\
\hline 21 & 0.052 & 11.42 & 105.03 & 56.91 & 32.23 & \\
\hline 22 & 0.044 & 9.86 & 102.40 & 57.02 & 35.22 & 4.94 \\
\hline 23 & 0.044 & 9.86 & 102.40 & 57.02 & 35.22 & 4.95 \\
\hline 24 & 0.044 & 9.86 & 102.40 & 57.02 & 35.22 & 4.95 \\
\hline \multicolumn{3}{|c|}{ Total } & 2130.85 & 937.58 & 440.03 & 14.85 \\
\hline
\end{tabular}

to the primary trajectory, adding about one year to the mission lifetime, but with minimal effect on other requirements on the spacecraft.

\subsection{Near-Sun Environment}

SPP will be exploring a region of space not yet explored-one potentially damaging to spacecraft and instruments. Surfaces of the spacecraft exposed to the Sun will experience about 475 Suns $\left(649 \mathrm{~kW} \mathrm{~m}^{-2}\right)$, or 475 times the solar irradiance experienced at Earth $\left(1366 \mathrm{~W} \mathrm{~m}^{-2}\right)$, at SPP's minimum perihelion. The high irradiance requires development and implementation of a thermal shield to enable the majority of the spacecraft systems and instruments to operate in a typical space thermal environment, and drives the thermal environment of the solar array and solar-exposed instruments.

At SPP's minimum perihelion, the Sun cannot be considered as a point source. SPP utilizes this aspect in the design of the solar arrays, exposing part of the solar arrays to only a fraction of the Sun (penumbra) at perihelion and protecting the remainder of the solar arrays in the umbra. This approach, along with the nearly $70^{\circ}$ wing incidence angle at minimum perihelion, reduces the irradiance on the solar arrays to about 25 Suns at end of life. SPP must account for the effects of the high nominal irradiance; higher off-nominal irradiance due to potential wing angle or spacecraft pointing anomalies; center-to-limb irradiance variations; non-uniform irradiance along a solar array string due to shadow-line to solar array string misalignments; as well as increased exposure to UV irradiance at elevated temperatures, resulting in increased solar array performance degradation.

The near Sun environment is accompanied by solar plasma and energetic charged particles, contributing to an intense radiation environment causing both spacecraft charging effects and radiation damage in materials and electronics. In addition, very small dust particles at high velocity in orbit around the Sun may impact the spacecraft; this concern has led to the performance of significant analyses and testing to accommodate the dust environment.

The above represents a few of the many environmental considerations unique to SPP. The SPP project science team has worked directly with the engineering team to provide in-depth 
Fig. 15 Anti-ram side of the SPP configuration, with instruments and significant components identified
Fig. 16 Ram side of the SPP configuration where most science instruments are mounted
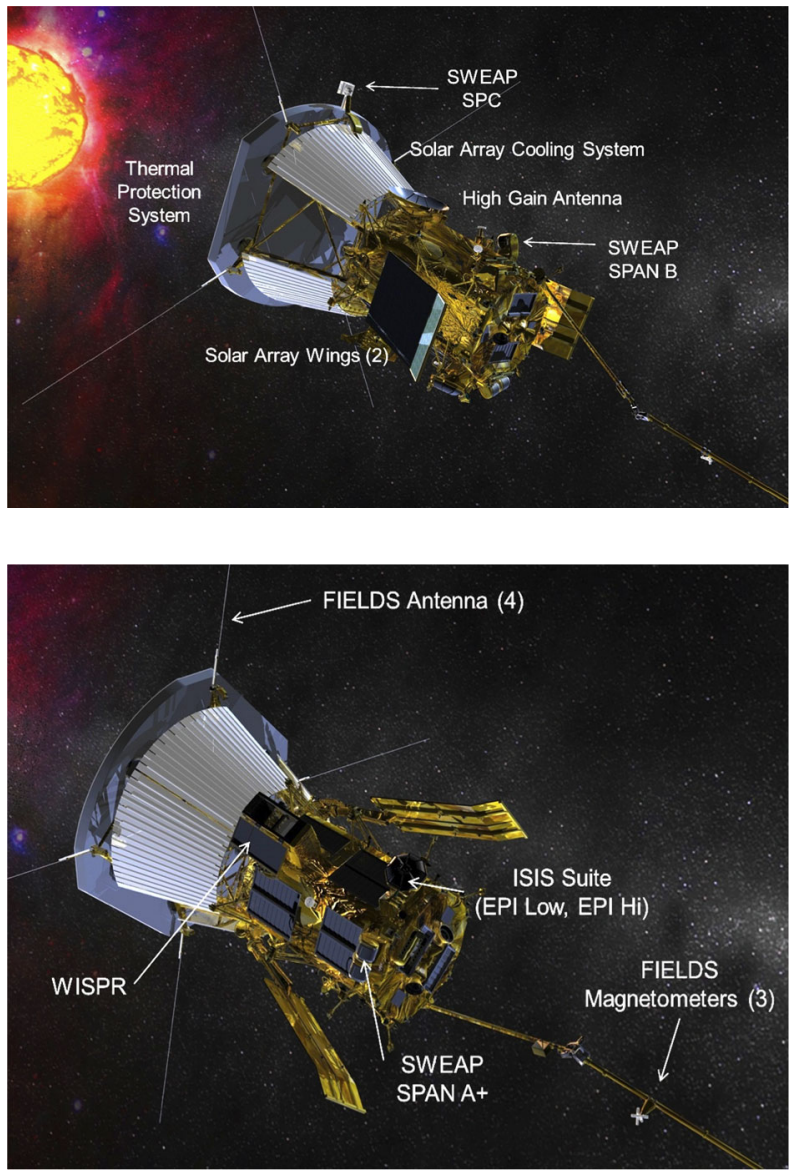

analyses and detailed models of the solar environments enabling the spacecraft design (e.g., Lario and Decker 2011; Lario 2012; Strong et al. 2015).

\subsection{SPP Spacecraft}

The SPP spacecraft, shown in Figs. 15, 16, is $685 \mathrm{~kg}$ (including propellant) at launch, approximately $3 \mathrm{~m}$ in height, and $2.3 \mathrm{~m}$ in diameter at the thermal protection system (TPS). The TPS is a carbon-carbon composite/carbon foam sandwich that makes the umbra to protect other spacecraft systems. For most spacecraft operations, the TPS is pointed sunward with only portions of the solar arrays, the SWEAP SPC (K2015), and the FIELDS Electric Field antennas (B2015) outside the umbra. When away from the Sun, the solar arrays are fully extended beyond the edges of the TPS with the cells pointed to the Sun. As the spacecraft approaches the Sun, the arrays are angled back increasing the flap angle so that the larger primary array is contained within the umbra and only the secondary array is illuminated. Although there is more than adequate solar flux for power generation at solar encounter, SPP power is limited to minimize the size of the active solar array cooling system (SACS), contributing to the ability of the spacecraft to meet the launch mass constraint.

The solar array for SPP is specifically designed to accommodate the high solar irradiance, both to maximize power production and to manage the thermal environment under nominal 
and off-nominal high irradiance conditions. The solar array strings are laid down, one string per solar array width, on an actively cooled titanium substrate, or platen. The solar cell stack is designed to efficiently conduct heat from the top of the cell through the stack to the platen. Water is pumped through channels in the titanium platen, up to the four cooling system primary radiators (CSPRs) mounted on the truss structure assembly (TSA) under the TPS, and back through the pump mounted on the top deck of the spacecraft. The cooling system is capable of dissipating $\sim 6500 \mathrm{~W}$ and maintains the solar array below $150^{\circ} \mathrm{C}$ at the solar cell stack to platen interface at minimum perihelion.

The spacecraft bus hexagonal structure provides an interface to the launch vehicle upper stage and to the TSA, as well as to the mechanisms for solar array and high gain antenna (HGA) deployment and positioning, the magnetometer boom, the majority of the spacecraft components and the science instruments. The mass constraint and the requirement to protect all components and instruments within the umbra created by the TPS result in tight spacecraft packaging.

The spacecraft thermal system uses louvers, blanketing, surface treatment, and heaters to maintain the spacecraft bus within nominal spacecraft operating conditions with minimal power consumption. Most spacecraft components are conductively coupled to the bus. Exceptions include the battery and most instruments, which are isolated from the spacecraft and include their own thermal control. Spacecraft components with large heat rejection requirements are packaged on the ram side and bottom deck panels, panels that are not exposed to the Sun except for specific time periods during launch and early operations far from the Sun. Although the spacecraft bus is protected from the harsh solar environment during encounter by the TPS, the range of thermal conditions that must be accommodated by the thermal design, including perihelion, aphelion, science data downlink slew, is significant.

The communication system provides the uplink, downlink and navigation service communication to Earth through NASA's Deep Space Network (DSN). The 0.6 m HGA, mounted on the anti-ram side of the spacecraft, provides high rate Ka-band science data downlink. Fanbeam antennas, also on the anti-ram side, are used for X-band uplink and lower rate housekeeping data downlink. Two low gain antennas, one on the ram side and one on the anti-ram side, provide near omni-directional coverage and are used primarily for launch and early operations, trajectory correction maneuvers, portions of solar encounter and emergency communication. Redundant X/Ka-band radios, redundant X-band and Kaband Traveling Wave Tube Amplifiers (TWTAs) are among the communication components packaged on the inside of the spacecraft bus.

The guidance and control (G\&C) system for the 3-axis controlled spacecraft maintains spacecraft attitude, necessary to meet science pointing requirements and also critical to maintain TPS pointing to the Sun protecting the spacecraft from the harsh solar environment inside of $0.7 \mathrm{AU}$. In addition, G\&C controls the solar array wing angles, HGA pointing, and executes propulsive maneuvers for spacecraft trajectory control. To accomplish this, $\mathrm{G} \& \mathrm{C}$ software algorithms run in the on-board flight computer, processing data and providing commands to the sensors and actuators. The G\&C sensors include redundant star trackers, mounted on the bottom deck, an internally redundant inertial measurement unit (IMU) mounted internal to the spacecraft, a sun sensor system including 7 solar limb sensors (SLS) distributed circumferentially around the spacecraft on brackets near the bottom deck, two digital sun sensors (DSS) on the anti-ram side of the spacecraft, and the corresponding electronics. The SLS provide an early warning of excursions from the intended Sun-pointing attitude. The DSS provides a direct measurement of solar location when the spacecraft is off-pointed from the Sun at solar distances greater than 0.7 AU to maintain pointing orientations supporting cooling system and spacecraft thermal constraints. Actuation is provided 
by four reaction wheels and the 12 thrusters in the blowdown hydrazine propulsion system. The thrusters are used both for delta-V maneuvers and for momentum wheel desaturation.

The avionics architecture maximizes the availability (even after a fault) of the sensors and actuators to $\mathrm{G} \& \mathrm{C}$ to ensure the spacecraft attitude control is maintained. The redundant processor module (RPM) includes three single board computers (SBC) a prime, hot spare and backup spare processor, and an avionics redundancy controller (ARC) that together with flight software (FSW) controls which physical processor performs which function and the transitions between them. The prime SBC executes flight software and operates the spacecraft. If a processor reset or fault occurs, for example, on the prime SBC, control is automatically switched to the hot spare, and the backup spare is promoted to hot spare. The original prime SBC becomes the backup spare, or if a fault has occurred and the SBC cannot be recovered, it is declared as failed. Even with a failed processor, the two remaining processors ensure G\&C control is maintained through a processor reset. Each SBC incorporates a solid-state recorder; science and spacecraft housekeeping data are recorded on the prime and hot spare SBC recorders. The avionics redundant electronics module (REM) incorporates a SpaceWire router for communication within the avionics subsystem, the radios, and the WISPR instrument, and provides interfaces to the spacecraft components and instruments. The avionics gathers critical spacecraft housekeeping telemetry at a high rate $(5 \mathrm{~Hz})$ to reduce the time to detect and resolve faults. The 16 remote interface units are distributed around the spacecraft and provide spacecraft and instrument temperature telemetry. The spacecraft also includes a power distribution unit (PDU) to provide switched, unswitched and pulsed power services.

The electrical power system includes the solar array, a $25 \mathrm{Ah}$ small cell lithium ion battery and the power system electronics (PSE), which provides the primary bus power to the PDU. The solar array flap wing angle is autonomously controlled and includes a power control mode to minimize thermal load to the cooling system while meeting spacecraft power requirements, a temperature control mode to ensure the cooling system is maintained above freezing conditions in nominal and fault conditions, and fixed angle modes. The wing angle control algorithms are developed by the power system team in conjunction with G\&C and implemented within the G\&C system. The battery supports the electrical load during launch, cooling system activation, the launch correction maneuver, Venus eclipse, small charge and discharge cycles during wing angle control, and fuse clearing in the case of a fault.

FIELDS, SWEAP, WISPR, and ISIS, are accommodated on the spacecraft, with the SWEAP SPAN A+, WISPR and ISIS EPI-Hi and EPI-Lo packaged on the spacecraft ram side (spacecraft $+x$ ); the SWEAP SPAN B packaged on the spacecraft anti-ram side; FIELDS Electric Fields antennas and SWEAP SPC packaged on the TSA near the TPS; and the FIELDS flux gate magnetometers, search coil, and V5 antenna integrated on the magnetometer boom (see Figs. 15, 16).

Protecting SPP from the harsh solar environment has been a primary design driver from the initial conception of the mission, and includes the following:

- The TPS coating and TPS foam thickness are sized to nearly eliminate the heat flow to the spacecraft and instruments from the 475 Suns environment closest approach, enabling the majority of SPP components and instruments to operate in a near standard spacecraft thermal environment.

- The TPS planform shape is defined such that spacecraft components and instruments are packaged within an $8^{\circ}$ packaging umbra on the ram and anti-ram side of the spacecraft and an $8.9^{\circ}$ packaging umbra on the $\pm y$ sides of the spacecraft, providing at least $2^{\circ}$ margin against the actual $5.82^{\circ}$ umbra at perihelion. G\&C is developed to meet a spacecraft 
pointing accuracy of $0.1^{\circ}$ under nominal conditions and under wheel control, well within the $2^{\circ}$ umbra margin.

- Solar Limb Sensors provide additional warning if there is ever a nearing umbra violation. Digital sun sensors on the anti-ram side of the spacecraft warn of anomalous attitudes when the spacecraft is intentionally off-pointed from the Sun. Sensor cells and temperature sensors on each solar array wing provide additional sensors to warn of high irradiance and/or over-temperature on the wings.

- The avionics architecture maximizes the availability (even after a fault) of sensors and actuators to guidance and control to ensure the spacecraft attitude control is maintained.

- The dust environment and dust impact analysis for the spacecraft and instruments has been developed in detail. Protection guidelines for the cooling system, spacecraft and instruments have been defined. The solar array wings are oriented parallel to the ram direction during encounter to minimize the probability of damage to the water-cooled solar array.

- The radiation environments have been developed and analyzed to provide high fidelity local environments for individual components, instruments, and the harness to support preliminary design. Worst-case proton events have been assessed to evaluate potential star tracker outage durations. Analyses of drift rate have defined IMU accuracy requirements, similar to those for the MESSENGER spacecraft, to ensure SPP remains within the umbra during these events.

- The spacecraft is designed to be single fault tolerant. Fault management is designed for autonomous safing and recovery.

- The mission design of successively reduced perihelia provides calibration opportunity in a less severe environment. As shown in Fig. 11, the first perihelion solar distance is $35.7 R_{S}$ as compared to the $<10 R_{S}$ perihelion for the final three orbits, resulting in lower heating of the TPS and solar array, a smaller umbra angle with larger umbra region, and lower sensitivity of the cooling system capacity to solar array flap angle.

\subsection{Technology Development}

Many of the SPP subsystems are based on previously flown technology. However, critical components were developed specifically for SPP, or were adapted and tested specifically for the SPP environments, a critical activity in the early phases of the project. Key technology development areas enabling the SPP mission are the thermal protection system (TPS), solar arrays, solar array cooling system, solar limb sensors, SWEAP SPC and FIELDS electric field antennas. Significant technology development was completed during Pre-Phase A, Phase A, and Phase B, such that each technology was at the necessary readiness level by Preliminary Design Review (PDR).

As described above, the SPP TPS is a lightweight, non-ablative, insulating layer that protects the spacecraft from the intense solar irradiance of $\sim 475$ Suns at $9.86 R_{S}$. The TPS consists of a carbon-carbon top and bottom facesheet with a carbon foam core, an alumina coating on the sun-facing surface, and features for attachment of the TPS to the supporting TSA. The planform shape provides two "knife edges" for the solar array wings, ensuring a uniform penumbra region for their operation, and four "chamfers" for 4 of the 7 solar limb sensors. The TPS technology development demonstrated the TPS performance in the thermal environment and in the launch environment. A full size TPS, shown in Fig. 17, was tested in the launch environment including vibration and acoustics, and a section of the TPS was fabricated and tested in thermal environments even more severe than the presumed thermal environment within $10 R_{S}$. In addition, the coating optical properties were demonstrated at beginning of life and end of life conditions of the mission. 
Fig. 17 Full-sized test model of the TPS mounted on a test TSA in preparation for acoustic testing at Goddard Space Flight Center's acoustic test facility

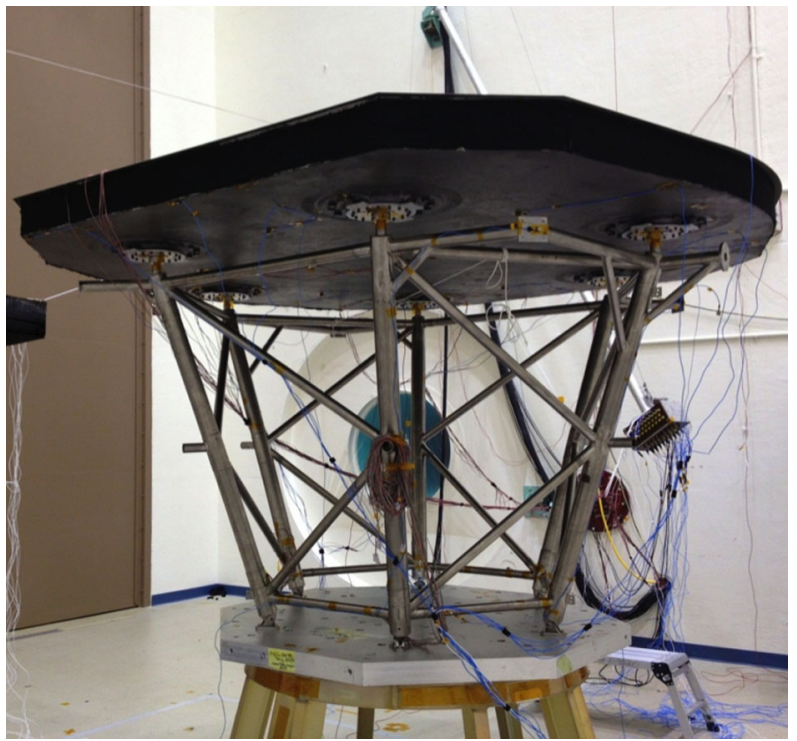

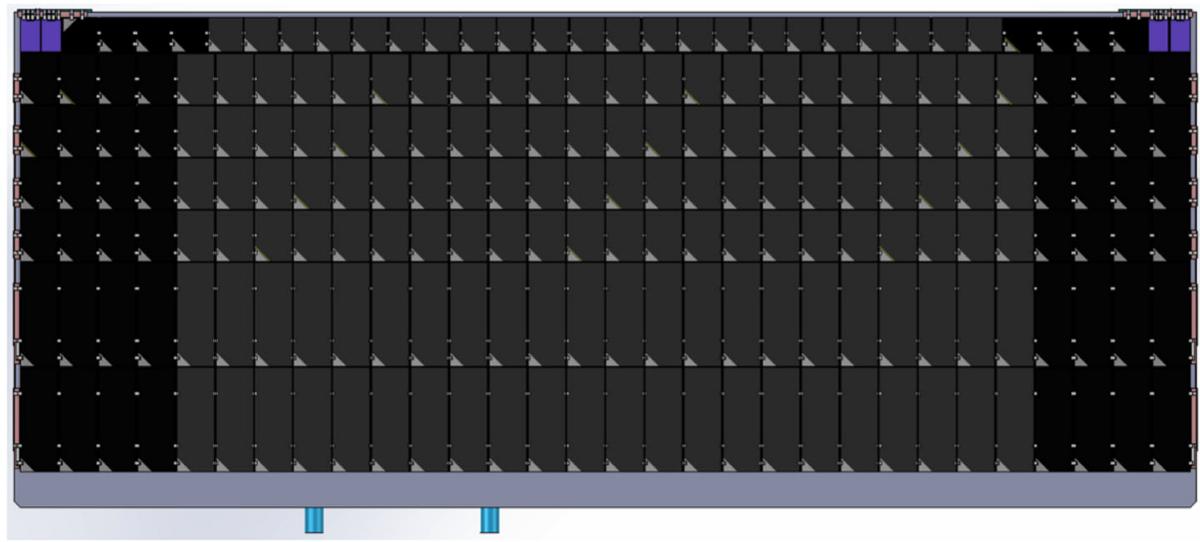

Fig. 18 Full-scale test panel of the secondary section of the Solar Array ready for environmental testing. This panel is $27 \mathrm{~cm} \times 65 \mathrm{~cm}$, not including the inlet and outlet manifolds for connection to the rest of the Solar Array Cooling System

Each of the two solar array wings has a primary and secondary section with a fixed cant angle between the two sections. Only the secondary section is illuminated at perihelion, reaching about 25 Suns on the outer-most string at end of life nominal conditions, so technology development efforts concentrated on this section. A full size secondary section, shown in Fig. 18, including a flight-like water-cooled substrate and flight-like solar cell assemblies was fabricated and successfully tested in relevant environments, including the high solar irradiance, high temperature nominal and off-nominal conditions.

The SACS dissipates the heat absorbed by solar array wings during closest approach to the Sun enabling the solar cells to operate within their temperature constraints while providing the required electrical power. In addition to dissipating $\sim 6500 \mathrm{~W}$ of heat at perihelion, the cooling system is designed and operated to prevent freezing at aphelion. Figure 19 shows 
Fig. 19 Full-scale, half size solar array cooling system test unit installed on a thermal-vacuum chamber for environmental testing. The dark radiator is visible; other components are hidden by MLI for thermal control during testing

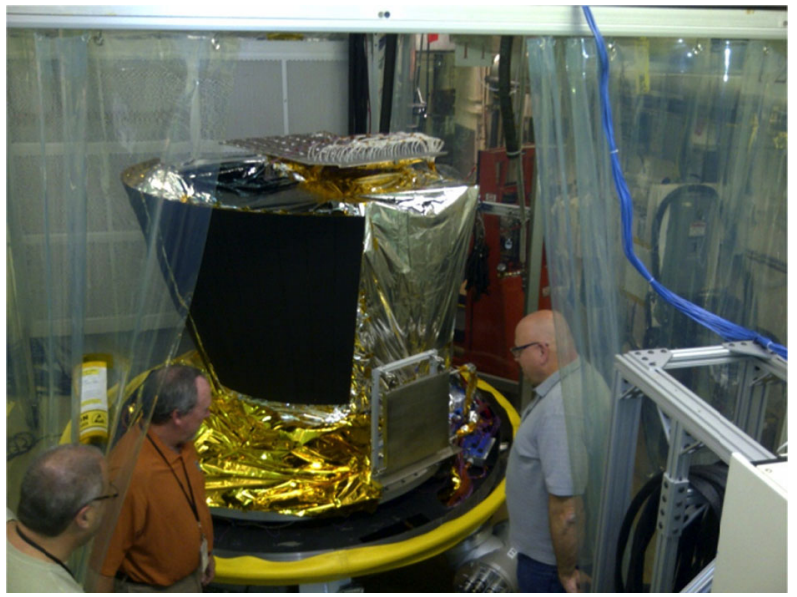

half of a full scale cooling system, including one solar array wing, two radiators and a pump, to demonstrate thermal and hydraulic performance at all driving operating conditions. An additional solar array wing and radiator were fabricated for structural testing, which was successfully completed.

Both the Faraday cup (SWEAP/SPC) (K2015) and the FIELDS electric field antennas (B2015) extend beyond the TPS and see the same environment as the TPS sunward-facing surface. The solar environment during solar encounter is significantly more severe than all previous experience with similar sensors. Therefore, technology development programs for each were implemented to demonstrate the operation of each in the expected SPP environment, and are described in the accompanying instrument papers (B2015, K2015).

\subsection{Mission Operations}

Spacecraft operations fall into two categories: science data collection, and cruise/downlink. Instrument measurements are mostly collected during the solar encounter phase of an orbit, with all instruments nominally on at $100 \%$ duty cycle during this phase. All instruments perform science measurements simultaneously, allowing for coordinated collection of data during interesting events ("burst mode") and a high degree of integration between science investigations. Detailed planning of each solar encounter is done for each orbit, coordinated by the SPP Science Working Group (SWG).

Given that communications are limited during the solar encounter phase, science data is stored on the solid-state recorders (SSRs) for downlink during the cruise/downlink phase of each orbit. The Sun-Probe-Earth geometry varies through the mission, and so opportunities for communication and downlink rates vary as well, requiring detailed planning unique to each orbit. In some cases, the downlink opportunities are insufficient to retrieve an entire orbit's data; the SSRs are sized to store data from two orbits to allow Mission Operations to balance downlink opportunities to return all data.

The cruise/downlink phase is also used for navigation contacts and trajectory correction maneuvers, as well as Venus gravity assists. Since the spacecraft is further away from the Sun during this phase, the thermal input from the Sun is significantly lower. Off-sun pointing attitudes are used between 0.7 and $0.76 \mathrm{AU}$ to enable the HGA to point to Earth for increased downlink opportunities. Outside of $0.82 \mathrm{AU}$, the spacecraft is pointed 45 degrees off-sun exposing the anti-ram side of the spacecraft to the Sun to maintain temperatures high enough 
to avoid freezing in the cooling system, and to reduce heater power requirements in the spacecraft and instruments. During this phase, instruments can be operated when power allows.

In addition to nominal spacecraft operations, a significant effort for the SPP team has been to develop a system capable of recovering from anomalous conditions before damage to spacecraft components from the solar environment occurs, especially in the cases of solar array control and spacecraft pointing excursions that could cause an umbra violation. Fault management incorporates analyses to identify potential failure modes, develops responses to each, and provides inputs to the development of rules and macros within the autonomy subsystem to implement responses. A large portion of the system testing prior to launch is devoted to showing that the system works as designed to detect and mitigate faults.

\section{SPP Science Operations}

The SPP mission uses a decoupled operations concept, which is implemented by distributing the Science Instrument Operations amongst the teams that developed the instruments. While the Mission Operations Team operates the spacecraft bus, the teams within the Science Operations Centers (SOC) operate their individual instruments independent of the bus operations and the operation of the other Instruments. The primary Mission Operation Center (MOC) functions include mission planning and scheduling, spacecraft command and control, and performance assessment. The MOC will be responsible for powering on and off the instruments. The SOC's instrument uplink and downlink operations are decoupled from spacecraft operations and operate independently from each other. The SOCs are responsible for the operation of their respective instruments. The SOC functions include planning, providing command sequences, monitoring their instrument's health and safety and production and archival of data products; details about the various SOCs are provided in the instrument papers (M2014, B2015, V2015, K2015).

SPP will use the DSN for communications. The instruments will generally not be powered on during the Ka-band downlink and some spacecraft activities for power considerations. The ability to close the link between the spacecraft and Earth is often constrained due to the line of sight being blocked by position of the Sun. The inability to downlink $100 \%$ of the science data on some orbits necessitates the accommodation of an solid-state recorder (SSR), which will hold the total data for two consecutive orbits.

\subsection{SPP Science Planning}

Orbit activity planning occurs in advance for each SPP orbit and includes downlink availability constraints on some of the orbits. The planning output products will be adjusted as more accurate inputs like DSN uplink and downlink periods and spacecraft ephemeris become available.

Multiple teams, the Orbit Planning Team (OPT), the SWG, and the individual instrument operations teams (IO team) perform the planning process. The OPT consists of personnel from various teams including mission operations (MOPS), spacecraft engineering, navigation, DSN, mission design, the Project Scientist and the instruments. The SWG consists of the Principal Investigators (PIs) and PI-designated representatives, and the Project and Mission Scientists. The SWG is responsible for the coordination and definition of the science activities, such as coordinated measurements, to perform during the orbit. 


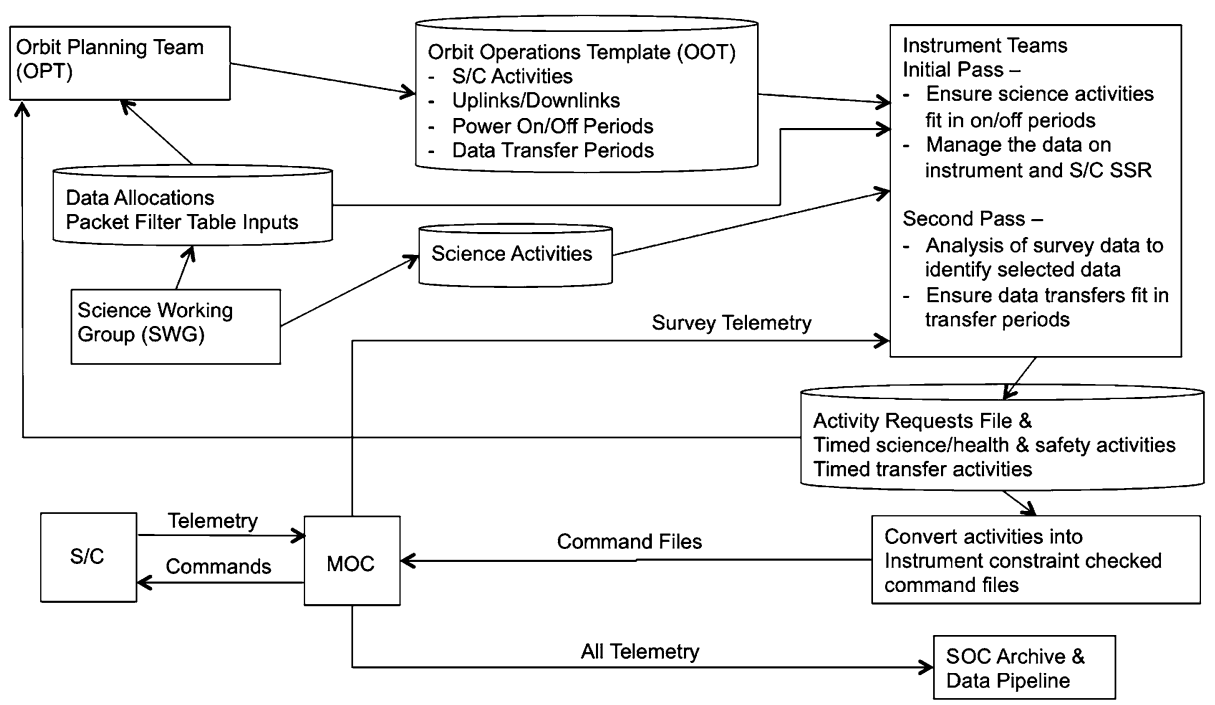

Fig. 20 Science Planning Process (boxes represent actions or items and cylinders represent files). The science planning process starts with the Orbit Planning Team (OPT), in the upper left, creating the Orbit Operations Template (OOT) files which the Instrument Teams, in the upper right, use along with the science activities in their science planning to produce activity requests. The activity requests are translated into command files and placed on the Mission Operation Center (MOC) where they are sent up to the spacecraft and on to the instrument. The activity requests also are sent back to the OPT. The planning process is cyclical in nature and updates are expected as inputs to the process change

Figure 20 describes the orbit planning process and its products, starting with the creation of an Orbit Operations Template (OOT) by the OPT approximately 3 months prior to the start of the orbit. The OOT is a group of products that include spacecraft activities, uplink and downlink periods, instrument power on and off periods and periods where instruments (FIELDS and SWEAP) can transfer data from their SSRs to the spacecraft SSR. The FIELDS and SWEAP instruments have internal SSRs with the ability to record more data than their respective data allocations. They will first downlink low-resolution "survey" data, which can be used to identify the high resolution data that they want to transfer to the spacecraft SSR for downlink. A science orbit-planning tool will be created to aid in the planning process.

The spacecraft flight software has a downlink priority system numbered 0 (highest) to 9 (lowest). High priority housekeeping for emergencies is sent as priority 0 . The spacecraft and instrument housekeeping are sent as priority 1, and the SWG will define the instruments' data allocations for priorities 2-9. This allows the SWG to build in flexibility in order to ensure the best downlink scheme is employed within the downlink constraints of any given orbit.

\subsection{Spacecraft-MOC-SOC Data Flow}

\subsubsection{Commanding}

Figure 21 contains a diagram of the command and data flow between the SOCs, MOC, spacecraft, and the instruments; the path of the commands from the SOC to the instrument is shown at the top. The SOCs will create a set of commands that implement the science 


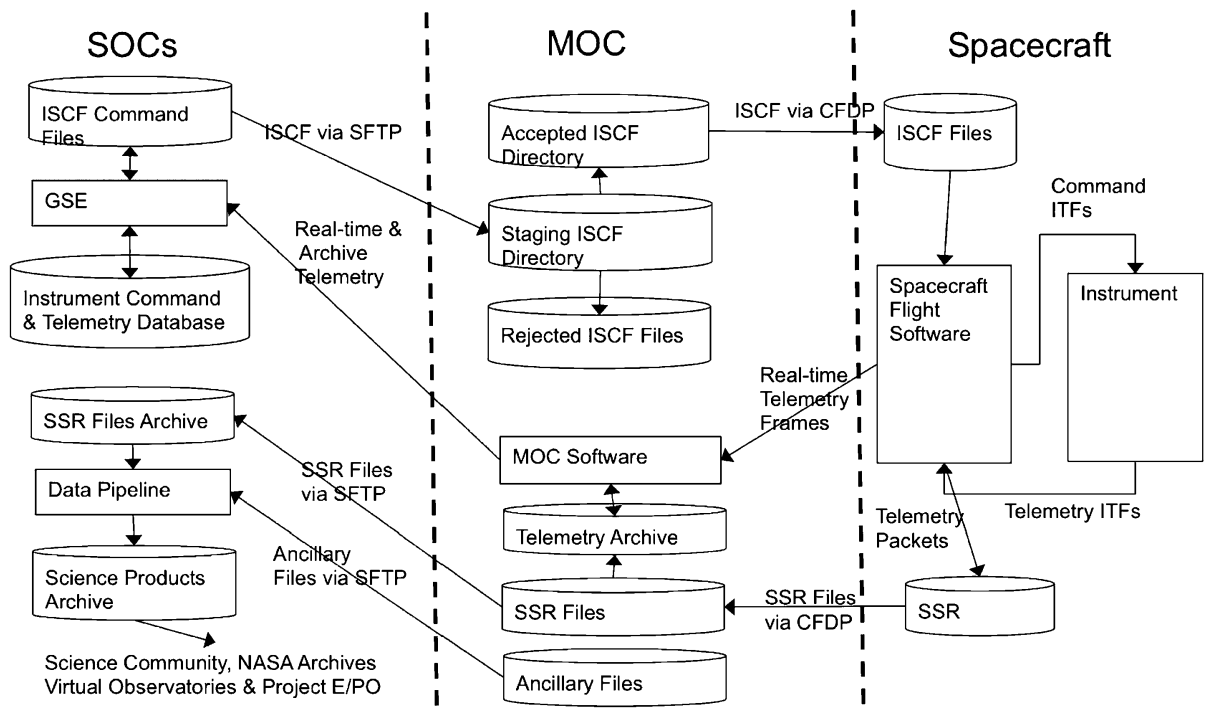

Fig. 21 SOC to Instrument Data Flow (boxes represent instruments or software systems and cylinders represent files). The data flow in the diagram starts in the upper left when the ISCF Command files are sent across to the MOC where they are validated and sent to the spacecraft and on to the instrument. The instrument sends telemetry ITF's which are packaged as real-time data and SSR files and are downlinked to the MOC. The SOC accesses the packet archive using their ground support equipment (GSE) or obtains the solid-state recorder (SSR) files using SFTP or rsync for their Data Pipeline

and instrument health and safety activities identified by their science and the instrument operation teams. The SOC's ground support equipment (GSE) is used to translate the list of commands into an Instrument Supplemented Command File (ISCF), which includes a command delivery time. The commands are validated and checked for instrument constraints by the individual instrument teams and are delivered to the MOC where file format validation is performed. Conflicts between team activity plans have been solved before this point through the use of a science planning tool and interactive dialog between the MOC and the instrument teams during the Science Planning process mentioned in the previous section.

The MOC uses the Consultative Committee for Space Data System (CCSDS) File Delivery Protocol (CFDP) to uplink the ISCFs. CFDP implements a 2-way handshaking between flight and ground software to ensure that intact files are received by the ground system. Should any file fragments be lost due to downlink dropouts, the handshaking results in retransmission of the missed fragments. On the spacecraft, the flight software dispatching commands via the Interface Transfer Frame (ITF) protocol to the designated instrument when it is on, and ready to accept commands.

\subsubsection{Data downlink}

During a scheduled contact with the DSN, the spacecraft will transmit real-time telemetry and the contents of the SSR to the ground. The SSR files are sent to the ground in priority order set by the packet filter table. SSR telemetry is forwarded to the MOC during contacts.

The MOC will produce files containing cleaned and merged Level 0 real-time telemetry for each instrument suite. The SOCs will use spacecraft attitude and other ancillary data available from the MOC for the production of the higher order science products. The MOC 
will maintain a disk-based archive containing both spacecraft and real-time instrument raw telemetry packets.

\subsubsection{Health and Safety}

The SOCs are responsible for insuring their instrument health and safety. They will monitor the instrument housekeeping telemetry stream and produce state-of-heath plots and data products based on the instrument housekeeping.

\subsubsection{Science Product Production}

The instrument SOC teams are responsible for the production of the science data products, which will typically be available as Common Data Format (CDF) or Flexible Image Transport System (FITS) files. Some products will require ancillary data products from the MOC such as spacecraft ephemeris and attitude and data from other SPP instruments. A summary of these products is given at a mission level in Table 4 .

The science products are made available across the SPP science team, to the science community, NASA archives, virtual observatories and to the project education and public outreach on an agreed upon production schedule and will be fully compliant with the NASA Heliophysics Science Data Management Policy.

The SPP mission will provide a Science Data Portal—a web-based interface providing a common point of entry of specific interest to SPP scientists as well as the general public. The SPP Science Data Portal will provide ancillary services, tools, data and links that benefit the SPP project itself and allow users from the wider heliophysics community to access SPP data and related products

\section{Summary and Conclusions}

The Solar Probe Plus mission is an extraordinary and historic mission. This mission will explore the most important region of the solar system to be visited by a spacecraft and will answer top-priority science questions that have remained unanswered since coronal heating was discovered in the 1930s and 1940s (Grotrian 1939; Saha 1942), and captured in the Simpson Committee report (1958). The space physics community has acknowledged the need for an extraordinary mission to provide detailed knowledge of the Sun to (1) Trace the flow of energy that heats the solar corona and accelerates the solar wind, (2) Determine the structure and dynamics of the plasma and magnetic fields at the sources of the solar wind, and (3) Explore the physical mechanisms that produce, accelerate, and transport energetic particles in the inner heliosphere. These critical advances can be achieved only through insitu measurements of the solar wind and energetic particles and close-by remote sensing imaging within the corona and have been of top priority in multiple Roadmaps and Decadal Surveys.

Calls for a "solar probe" actually predate NASA, but only recently has our technology caught up with this critical scientific need. SPP will explore the inner heliosphere region, traveling much closer to the Sun than any other spacecraft, to determine the structure and dynamics of the Sun's coronal magnetic field, understand how the corona and solar wind are heated and accelerated, and how energetic particles are produced and their distributions evolve. Thus, SPP will finally answer the fundamental questions, and provide transformational science results. 
Table 4 Mission Level Data Products. A mission-level summary of the science data products delivered by the instrument SOC teams, by data level. Quick look data will be available after a grace period of 6 months after downlink of all data and ancillary data sources for an encounter for the first two orbits, and within 60 days of downlink for the following orbits. Final data will be released no later than 6 months from downlink of all data from an encounter and receipt of all ancillary data sources

\begin{tabular}{|c|c|c|}
\hline $\begin{array}{l}\text { Data } \\
\text { level }\end{array}$ & Instrument & Description \\
\hline \multirow[t]{7}{*}{ L0 } & FIELDS & Raw telemetry produced by SPP MOC. \\
\hline & & Possibly 24-hour, ApID-separated, sorted, cleaned. \\
\hline & & PTP and SSR binary files. \\
\hline & SWEAP & Raw Telemetry \\
\hline & & (Raw de-commutated telemetry received from MOC). \\
\hline & ISIS & Raw telemetry packets, including $\mathrm{HK}$, Cmd-response, rates, and events packets. \\
\hline & WISPR & Raw telemetry (CCSDS data packets). \\
\hline \multirow[t]{7}{*}{ L1 } & FIELDS & $\begin{array}{l}\text { Uncompressed and decommutated } \mathrm{LO}+\text { Time-tagged waveform and spectral } \\
\text { data in telemetry and engineering units }[\mathrm{V}, \mathrm{dBs}, \mathrm{nT}] \text { in spacecraft coordinate } \\
\text { system. Data affinity groups (CDFs). }\end{array}$ \\
\hline & & Quick Look and daily/orbital summary plots. \\
\hline & SWEAP & Instrument Count Rates (SPANs). \\
\hline & & Instrument Currents (SPC). \\
\hline & ISIS & $\begin{array}{l}\text { Time series of uncalibrated instrument science and engineering rates at highest } \\
\text { resolution. }\end{array}$ \\
\hline & & Unpacked particle event data. \\
\hline & WISPR & FITS files with uncompressed images. Image values are in raw counts (DN). \\
\hline
\end{tabular}

L2 FIELDS L1 + Time-tagged waveform and spectral data in fully calibrated physical units $[\mathrm{V}, \mathrm{mV} / \mathrm{m}, \mathrm{nT},(\mathrm{V} / \mathrm{m}) 2 / \mathrm{Hz}, \mathrm{nT} 2 / \mathrm{Hz}]$ in spacecraft and heliophysical coordinate systems (CDFs).

Quick Look and daily/orbital summary plots.

SWEAP Calibrated Particle flux (Calibrated and in physical coordinates and units). Solar Wind moments and energy spectra (Calculated onboard, calibrated, in physical coordinates and units).

ISIS Time series of calibrated particle intensities at highest time, energy, and look-direction resolution, in physical units.

WISPR FITS files with calibrations applied. Image values in units of brightness.

L3 FIELDS

L2 + VxB removal for DC E-field measurement, offsets and corrections with data quality flags.

Plasma density. Spacecraft potential. Merged B.

Merged density and temperature (FIELDS-SWEAP).

CDFs, Science data plots.

SWEAP Solar wind bulk parameters, energy spectra, and electron pitch angle distribution (Calibrated and calculated on the ground).

ISIS Time series of calibrated particle intensities, averaged into appropriate sets of larger time, energy and look-direction bins.

WISPR Data products are the result of combining two or more images (movies, Carrington maps, etc). May or may not be calibrated in physical units.

L4 FIELDS

Event (shocks, current sheets, radio bursts, stream interaction regions) time tags and parameters.

SWEAP Derived power spectra, source location, and event lists.

ISIS Particle spectra and fluences for specific events and/or periods.

Particle anisotropy parameters/plots.

WISPR Derived quantities (electron densities, CME masses). 
The SPP mission will accomplish this using seven Venus encounters to gradually lower the perihelion from $35 R_{S}$ to within $10 R_{S}$ over 24 orbits, traveling close enough to the Sun to provide the critical data, but not so close to preclude implementation within the available resources. Repeatedly flying through the region where the solar wind is accelerated, SPP will measure, with the required cadence and sensitivity, the magnetic and electric fields and their fluctuations and variations, velocity distribution functions to derive the properties of the thermal solar wind plasma, the intensity, spectrum and pitch-angle distribution of energetic electrons, protons and heavy ions, and broadband visible light observations. SPP will be able to finally address the critical science questions detailed above by providing the key insitu and remote sensing measurements, which are currently lacking, closer to the Sun than any other spacecraft has journeyed. With the data it transmits back to Earth, solar and space physicists will answer questions that cannot be answered by any other means and will attain a deep understanding of phenomena and processes in this fascinating and critical region.

But SPP is more than a fundamental physics mission. The physics of the corona and inner heliosphere connect the activity of the Sun to the environment and technological infrastructure of the Earth. Events on the Sun drive the fundamental physics of the heliosphere, aurora, and magnetosphere of the Earth and other planets, affecting satellite communications, power grids, pipelines, radiation exposure on airline flights, and astronaut safety. The effect of solar activity can be traced up to the heliospheric termination shock and maybe into the interstellar medium. Until we can explain the physical processes occurring close to the Sun, we will not be able to accurately predict space weather effects that can cause havoc at Earth. In order to unlock the mysteries of the corona, but also to protect a society that is increasingly dependent on technology from the threats of space weather, we will send Solar Probe Plus to touch the Sun.

Finally, as with any great voyage into uncharted realms, SPP's journey to the Sun holds the promise of many more unanticipated discoveries - new mysteries to challenge humankind's ever-expanding knowledge of our home in the universe.

Acknowledgements The Solar Probe Plus mission is the result of tireless efforts by countless scientists from all over the world who first imagined this project in the 1950s and never ceased in their efforts over the following decades to ensure that the mission will finally become a reality. We are deeply indebted to all of them for their outstanding work and remarkable determination and vision. We acknowledge the continuous support of NASA to the project. Solar Probe Plus is part of NASA's Living With a Star program. We, the authors, acknowledge the support from NASA under contract NNN06AA01C (Task NNN10AA08T). We would like also to acknowledge the leadership and friendship of the late Andrew Dantzler (the first Solar Probe Plus Project Manager). We are very grateful to the anonymous referees for the constructive criticism that helped improve the quality of the manuscript.

Open Access This article is distributed under the terms of the Creative Commons Attribution 4.0 International License (http://creativecommons.org/licenses/by/4.0/), which permits unrestricted use, distribution, and reproduction in any medium, provided you give appropriate credit to the original author(s) and the source, provide a link to the Creative Commons license, and indicate if changes were made.

\section{References}

M.D. Altschuler, G. Newkirk, Magnetic fields and the structure of the solar corona. I: methods of calculating coronal fields. Sol. Phys. 9, 131-149 (1969). doi:10.1007/BF00145734

T. Antecki, R. Schlickeiser, M. Zhang, Stochastic acceleration of suprathermal particles under pressure balance conditions. Astrophys. J. 764, 89 (2013). doi:10.1088/0004-637X/764/1/89

S.K. Antiochos, Z. Mikic, R. Lionello, V. Titov, J. Linker, A model for the sources of the slow solar wind, in American Astronomical Society Meeting Abstracts \#216. Bulletin of the American Astronomical Society, vol. 41 (2010), pp. 405-421 
S.K. Antiochos, J.A. Linker, R. Lionello, Z. Mikić, V. Titov, T.H. Zurbuchen, The structure and dynamics of the corona-heliosphere connection. Space Sci. Rev. 172, 169-185 (2012). doi:10.1007/s11214011-9795-7

M. Asgari-Targhi, A.A. van Ballegooijen, S.R. Cranmer, E.E. DeLuca, The spatial and temporal dependence of coronal heating by Alfvén wave turbulence. Astrophys. J. 773, 111 (2013). doi:10.1088/0004-637X/ $773 / 2 / 111$

W.I. Axford, J.F. McKenzie, The origin of high speed solar wind streams, in Solar Wind Seven Colloquium, ed. by E. Marsch, R. Schwenn (1992), pp. 1-5

S.D. Bale, J.C. Kasper, G.G. Howes, E. Quataert, C. Salem, D. Sundkvist, Magnetic fluctuation power near proton temperature anisotropy instability thresholds in the solar wind. Phys. Rev. Lett. 103(21), 211101 (2009). doi:10.1103/PhysRevLett.103.211101

S.D. Bale, K. Goetz, P.R. Harvey et al., The FIELDS instrument suite for Solar Probe Plus: measuring the coronal plasma and magnetic field, plasma waves and turbulence, and radio signatures of solar transients. Space Sci. Rev. (2015, this issue). doi:10.1007/s11214-014-0109-8

A. Balogh, R.J. Forsyth, E.A. Lucek, T.S. Horbury, E.J. Smith, Heliospheric magnetic field polarity inversions at high heliographic latitudes. Geophys. Res. Lett. 26, 631-634 (1999). doi:10.1029/1999GL900061

L. Bettarini, S. Landi, F.A. Rappazzo, M. Velli, M. Opher, Tearing and Kelvin-Helmholtz instabilities in the heliospheric plasma. Astron. Astrophys. 452, 321-330 (2006). doi:10.1051/0004-6361:20054285

B. Breech, W.H. Matthaeus, J. Minnie, J.W. Bieber, S. Oughton, C.W. Smith, P.A. Isenberg, Turbulence transport throughout the heliosphere. J. Geophys. Res. Space Phys. 113, 8105 (2008). doi:10.1029/ 2007JA012711

R. Bruno, V. Carbone, The solar wind as a turbulence laboratory. Living Rev. Sol. Phys. 2, 4 (2005). doi: 10.12942/lrsp-2005-4

R. Bruno, V. Carbone, The solar wind as a turbulence laboratory. Living Rev. Sol. Phys. 10, 2 (2013). doi: 10.12942/lrsp-2013-2

L. Burlaga, D. Berdichevsky, N. Gopalswamy, R. Lepping, T. Zurbuchen, Merged interaction regions at 1 AU. J. Geophys. Res. Space Phys. 108, 1425 (2003). doi:10.1029/2003JA010088

S.R. Cranmer, An efficient approximation of the coronal heating rate for use in global sun-heliosphere simulations. Astrophys. J. 710, 676-688 (2010). doi:10.1088/0004-637X/710/1/676

S.R. Cranmer, A.A. van Ballegooijen, R.J. Edgar, Self-consistent coronal heating and solar wind acceleration from anisotropic magnetohydrodynamic turbulence. Astrophys. J. Suppl. Ser. 171, 520-551 (2007). doi:10.1086/518001

C.E. Deforest, J.T. Hoeksema, J.B. Gurman et al., Polar plume anatomy: results of a coordinated observation. Sol. Phys. 175, 393-410 (1997). doi:10.1023/A:1004955223306

C.E. DeForest, P.L. Lamy, A. Llebaria, Solar polar plume lifetime and coronal hole expansion: determination from long-term observations. Astrophys. J. 560, 490-498 (2001). doi:10.1086/322497

J.F. Drake, M. Swisdak, Ion heating and acceleration during magnetic reconnection relevant to the corona. Space Sci. Rev. 172, 227-240 (2012). doi:10.1007/s11214-012-9903-3

W. Dröge, Y.Y. Kartavykh, B. Klecker, G.A. Kovaltsov, Anisotropic three-dimensional focused transport of solar energetic particles in the inner heliosphere. Astrophys. J. 709, 912-919 (2010). doi:10.1088/0004$637 \mathrm{X} / 709 / 2 / 912$

W.C. Feldman, S.R. Habbal, G. Hoogeveen, Y.-M. Wang, Experimental constraints on pulsed and steady state models of the solar wind near the Sun. J. Geophys. Res. 102, 26905-26918 (1997). doi:10.1029/ 97JA02436

L.A. Fisk, The acceleration of energetic particles in the interplanetary medium by transit time damping. J. Geophys. Res. 81, 4633-4640 (1976). doi:10.1029/JA081i025p04633

L.A. Fisk, Motion of the footpoints of heliospheric magnetic field lines at the Sun: implications for recurrent energetic particle events at high heliographic latitudes. J. Geophys. Res. 101, 15547-15554 (1996). doi:10.1029/96JA01005

L.A. Fisk, G. Gloeckler, The common spectrum for accelerated ions in the quiet-time solar wind. Astrophys. J. Lett. 640, 79-82 (2006). doi:10.1086/503293

L.A. Fisk, G. Gloeckler, Particle acceleration in the heliosphere: implications for astrophysics. Space Sci. Rev. 173, 433-458 (2012). doi:10.1007/s11214-012-9899-8

J. Geiss, G. Gloeckler, R. von Steiger, Origin of the solar wind from composition data. Space Sci. Rev. 72, 49-60 (1995). doi:10.1007/BF00768753

J. Giacalone, The acceleration of inner-source pickup ions by a propagating interplanetary shock, in American Institute of Physics Conference Series, ed. by J. Le Roux, G.P. Zank, A.J. Coates, V. Florinski American Institute of Physics Conference Series, vol. 1302 (2010), pp. 119-124. doi:10.1063/1.3529958

J. Giacalone, J.R. Jokipii, J. Kóta, Particle acceleration in solar wind compression regions. Astrophys. J. 573, 845-850 (2002). doi:10.1086/340660 
G. Gloeckler, In-situ detection of energetic particles, in Heliophysics, Space Storms and Radiation: Causes and Effects, ed. by C.J. Schrijver, G.L. Siscoe (Cambridge University Press, Cambridge, 2010), p. 43

G. Gloeckler, T.H. Zurbuchen, J. Geiss, Implications of the observed anticorrelation between solar wind speed and coronal electron temperature. J. Geophys. Res. Space Phys. 108, 1158 (2003). doi:10.1029/ 2002JA009286

M.L. Goelzer, N.A. Schwadron, C.W. Smith, An analysis of Alfvén radius based on sunspot number from 1749 to today. J. Geophys. Res. Space Phys. 119, 115-120 (2014). doi:10.1002/2013JA019420

J.T. Gosling, Observations of magnetic reconnection in the turbulent high-speed solar wind. Astrophys. J. Lett. 671, 73-76 (2007). doi:10.1086/524842

R.R. Grall, W.A. Coles, M.T. Klinglesmith, Observations of the solar wind speed near the Sun, in American Institute of Physics Conference Series, vol. 382, ed. by D. Winterhalter, J.T. Gosling, S.R. Habbal, W.S. Kurth, M. Neugebauer (1996), p. 108. doi:10.1063/1.51353

W. Grotrian, Zur Frage der Deutung der Linien im Spektrum der Sonnenkorona. Naturwissenschaften 27, 214 (1939). doi:10.1007/BF01488890

P. Hellinger, P. Trávníček, J.C. Kasper, A.J. Lazarus, Solar wind proton temperature anisotropy: linear theory and WIND/SWE observations. Geophys. Res. Lett. 33, 9101 (2006). doi:10.1029/2006GL025925

T.S. Horbury, M. Forman, S. Oughton, Anisotropic scaling of magnetohydrodynamic turbulence. Phys. Rev. Lett. 101(17), 175005 (2008). doi:10.1103/PhysRevLett.101.175005

P.A. Isenberg, Evolution of interstellar pickup ions in the solar wind. J. Geophys. Res. 92, 1067-1073 (1987). doi:10.1029/JA092iA02p01067

J.R. Jokipii, Deceleration and acceleration of cosmic rays in the solar wind. Phys. Rev. Lett. 26, 666-669 (1971). doi:10.1103/PhysRevLett.26.666

J.R. Jokipii, M.A. Lee, Compression acceleration in astrophysical plasmas and the production of $f(v) \propto v^{-5}$ spectra in the heliosphere. Astrophys. J. 713, 475-483 (2010). doi:10.1088/0004-637X/713/1/475

S.I. Jones, J.M. Davila, Localized plasma density enhancements observed in STEREO COR1. Astrophys. J. 701, 1906-1910 (2009). doi:10.1088/0004-637X/701/2/1906

S. Kahler, Injection profiles of solar energetic particles as functions of coronal mass ejection heights. Astrophys. J. 428, 837-842 (1994). doi:10.1086/174292

J.C. Kasper, A.J. Lazarus, S.P. Gary, Wind/SWE observations of firehose constraint on solar wind proton temperature anisotropy. Geophys. Res. Lett. 29, 1839 (2002). doi:10.1029/2002GL015128

J.C. Kasper, B.A. Maruca, M.L. Stevens, A. Zaslavsky, Sensitive test for ion-cyclotron resonant heating in the solar wind. Phys. Rev. Lett. 110(9), 091102 (2013). doi:10.1103/PhysRevLett.110.091102

J.C. Kasper, R. Abiad, G. Austin et al., Solar wind electrons alphas and protons (SWEAP) investigation: design of the solar wind and coronal plasma instrument suite for solar probe plus. Space Sci. Rev. (2015, this issue). doi:10.1007/s11214-015-0206-3

V. Katsikas, G. Exarhos, X. Moussas, Study of the solar Slow Sonic, Alfvén and Fast Magnetosonic transition surfaces. Adv. Space Res. 46, 382-390 (2010). doi:10.1016/j.asr.2010.05.003

C.F. Kennel, F.L. Scarf, F.V. Coroniti et al., Plasma and energetic particle structure upstream of a quasiparallel interplanetary shock. J. Geophys. Res. 89, 5419-5435 (1984). doi:10.1029/JA089iA07p05419

Y.-K. Ko, J.C. Raymond, T.H. Zurbuchen, P. Riley, J.M. Raines, L. Strachan, Abundance variation at the vicinity of an active region and the coronal origin of the slow solar wind. Astrophys. J. 646, 1275-1287 (2006). doi:10.1086/505021

S. Landi, P. Hellinger, M. Velli, Heliospheric magnetic field polarity inversions driven by radial velocity field structures. Geophys. Res. Lett. 33, 14101 (2006). doi:10.1029/2006GL026308

D. Lario, Estimation of the solar flare neutron worst-case fluxes and fluences for missions traveling close to the Sun. Space Weather 10, 3002 (2012). doi:10.1029/2011SW000732

D. Lario, R.B. Decker, Estimation of solar energetic proton mission-integrated fluences and peak intensities for missions traveling close to the Sun. Space Weather 9, 11003 (2011). doi:10.1002/2011SW000708

M.A. Lee, Coupled hydromagnetic wave excitation and ion acceleration at an evolving coronal/interplanetary shock. Astrophys. J. Suppl. Ser. 158, 38-67 (2005). doi:10.1086/428753

M.A. Lee, R.A. Mewaldt, J. Giacalone, Shock acceleration of ions in the heliosphere. Space Sci. Rev. 173, 247-281 (2012). doi:10.1007/s11214-012-9932-y

R.P. Lin, K.A. Anderson, S. Ashford et al., A three-dimensional plasma and energetic particle investigation for the wind spacecraft. Space Sci. Rev. 71, 125-153 (1995). doi:10.1007/BF00751328

R. Lionello, M. Velli, C. Downs, J.A. Linker, Z. Mikić, A. Verdini, Validating a time-dependent turbulencedriven model of the solar wind. Astrophys. J. 784, 120 (2014). doi:10.1088/0004-637X/784/2/120

M.K. Lockwood, J. Kinnison, N. Fox, R. Conde, A. Driesman, Solar probe plus mission definition, in Proceedings of the 63rd International Astronautical Congress (IAF, Naples, 2012)

J.W. Lynn, E. Quataert, B.D.G. Chandran, I.J. Parrish, The efficiency of second-order Fermi acceleration by weakly compressible magnetohydrodynamic turbulence. Astrophys. J. 777, 128 (2013). doi:10.1088/ 0004-637X/777/2/128 
M. Maksimovic, V. Pierrard, P. Riley, Ulysses electron distributions fitted with Kappa functions. Geophys. Res. Lett. 24, 1151-1154 (1997). doi:10.1029/97GL00992

M. Maksimovic, I. Zouganelis, J.-Y. Chaufray et al., Radial evolution of the electron distribution functions in the fast solar wind between 0.3 and 1.5 AU. J. Geophys. Res. Space Phys. 110, 9104 (2005). doi:10.1029/2005JA011119

F. Malara, M. Velli, Parametric instability of a large-amplitude nonmonochromatic Alfvén wave. Phys. Plasmas 3, 4427-4433 (1996). doi:10.1063/1.872043

F. Malara, L. Primavera, P. Veltri, Dissipation of Alfvén waves in compressible inhomogeneous media. Nuovo Cimento C 20, 903-909 (1997)

E. Marsch, C.Y. Tu, Non-Gaussian probability distributions of solar wind fluctuations. Ann. Geophys. 12, 1127-1138 (1994). doi:10.1007/s00585-994-1127-8

E. Marsch, C.-Y. Tu, The effects of high-frequency Alfvén waves on coronal heating and solar wind acceleration. Astron. Astrophys. 319, 17-20 (1997)

L. Matteini, S. Landi, P. Hellinger et al., Evolution of the solar wind proton temperature anisotropy from 0.3 to 2.5 AU. Geophys. Res. Lett. 34, 20105 (2007). doi:10.1029/2007GL030920

L. Matteini, P. Hellinger, S. Landi, P.M. Trávníček, M. Velli, Ion kinetics in the solar wind: coupling global expansion to local microphysics. Space Sci. Rev. 172, 373-396 (2012). doi:10.1007/s11214-011-9774-Z

L. Matteini, P. Hellinger, B.E. Goldstein et al., Signatures of kinetic instabilities in the solar wind. J. Geophys. Res. Space Phys. 118, 2771-2782 (2013). doi:10.1002/jgra.50320

L. Matteini, T.S. Horbury, M. Neugebauer, B.E. Goldstein, Dependence of solar wind speed on the local magnetic field orientation: role of Alfvénic fluctuations. Geophys. Res. Lett. 41, 259-265 (2014). doi:10.1002/2013GL058482

W.H. Matthaeus, G.P. Zank, S. Oughton, D.J. Mullan, P. Dmitruk, Coronal heating by magnetohydrodynamic turbulence driven by reflected low-frequency waves. Astrophys. J. Lett. 523, 93-96 (1999). doi:10.1086/312259

D.J. McComas, S.J. Bame, B.L. Barraclough et al., Ulysses' return to the slow solar wind. Geophys. Res. Lett. 25, 1-4 (1998). doi:10.1029/97GL03444

D.J. McComas, H.A. Elliott, N.A. Schwadron et al., The three-dimensional solar wind around solar maximum. Geophys. Res. Lett. 30, 1517 (2003). doi:10.1029/2003GL017136

D.J. McComas, M. Velli, W.S. Lewis et al., Understanding coronal heating and solar wind acceleration: case for in situ near-Sun measurements. Rev. Geophys. 45, 1004 (2007). doi:10.1029/2006RG000195

D.J. McComas, N. Angold, H.A. Elliott et al., Weakest solar wind of the space age and the current "mini" solar maximum. Astrophys. J. 779, 2 (2013). doi:10.1088/0004-637X/779/1/2

D.J. McComas, N. Alexander, N. Angold et al., Integrated Science Investigation of the Sun (ISIS): design of the energetic particle investigation. Space Sci. Rev. (2014). doi:10.1007/s11214-014-0059-1

R.A. Mewaldt, Solar energetic particle composition, energy spectra, and space weather. Space Sci. Rev. 124, 303-316 (2006). doi:10.1007/s11214-006-9091-0

R.A. Mewaldt, M.D. Looper, C.M.S. Cohen et al., Energy spectra, composition, and other properties of ground-level events during solar cycle 23. Space Sci. Rev. 171, 97-120 (2012). doi:10.1007/s11214012-9884-2

M. Neugebauer, P.C. Liewer, Creation and destruction of transitory coronal holes and their fast solar wind streams. J. Geophys. Res. Space Phys. 108, 1013 (2003). doi:10.1029/2002JA009326

M. Neugebauer, B.E. Goldstein, D.J. McComas, S.T. Suess, A. Balogh, Ulysses observations of microstreams in the solar wind from coronal holes. J. Geophys. Res. 100, 23389-23396 (1995). doi: 10.1029/95JA02723

N.S. Padhye, C.W. Smith, W.H. Matthaeus, Distribution of magnetic field components in the solar wind plasma. J. Geophys. Res. 106, 18635-18650 (2001). doi:10.1029/2000JA000293

S. Parhi, S.T. Suess, M. Sulkanen, Can Kelvin-Helmholtz instabilities of jet-like structures and plumes cause solar wind fluctuations at 1 AU? J. Geophys. Res. 104, 14781-14788 (1999). doi:10.1029/ 1999JA900041

E.N. Parker, Topological dissipation and the small-scale fields in turbulent gases. Astrophys. J. 174, 499 (1972). doi:10.1086/151512

E.N. Parker, Heating solar coronal holes. Astrophys. J. 372, 719-727 (1991). doi:10.1086/170015

J.C. Perez, B.D.G. Chandran, Direct numerical simulations of reflection-driven, reduced magnetohydrodynamic turbulence from the Sun to the Alfvén Critical Point. Astrophys. J. 776, 124 (2013). doi:10.1088/ 0004-637X/776/2/124

W.G. Pilipp, K.-H. Muehlhaeuser, H. Miggenrieder, M.D. Montgomery, H. Rosenbauer, Characteristics of electron velocity distribution functions in the solar wind derived from the HELIOS plasma experiment. J. Geophys. Res. 92, 1075-1092 (1987). doi:10.1029/JA092iA02p01075

J.J. Podesta, Dependence of solar-wind power spectra on the direction of the local mean magnetic field. Astrophys. J. 698, 986-999 (2009). doi:10.1088/0004-637X/698/2/986 
B.M. Randol, E.R. Christian, Simulations of plasma obeying Coulomb's law and the formation of suprathermal ion tails in the solar wind. J. Geophys. Res. Space Phys. 119, 7025-7037 (2014). doi:10.1002/ 2014JA020095

N.E. Raouafi, G. Stenborg, Role of transients in the sustainability of solar coronal plumes. Astrophys. J. 787, 118 (2014). doi:10.1088/0004-637X/787/2/118

N.E. Raouafi, J.W. Harvey, S.K. Solanki, Properties of solar polar coronal plumes constrained by ultraviolet coronagraph spectrometer data. Astrophys. J. 658, 643-656 (2007). doi:10.1086/510286

N.E. Raouafi, G.J.D. Petrie, A.A. Norton, C.J. Henney, S.K. Solanki, Evidence for polar jets as precursors of polar plume formation. Astrophys. J. Lett. 682, 137-140 (2008). doi:10.1086/591125

A.F. Rappazzo, E.N. Parker, Current sheets formation in tangled coronal magnetic fields. Astrophys. J. Lett. 773, 2 (2013). doi:10.1088/2041-8205/773/1/L2

A.F. Rappazzo, M. Velli, G. Einaudi, R.B. Dahlburg, Diamagnetic and expansion effects on the observable properties of the slow solar wind in a coronal streamer. Astrophys. J. 633, 474-488 (2005). doi:10.1086/ 431916

J.C. Raymond, S. Krucker, R.P. Lin, V. Petrosian, Observational aspects of particle acceleration in large solar flares. Space Sci. Rev. 173, 197-221 (2012). doi:10.1007/s11214-012-9897-x

D.V. Reames, Particle acceleration at the Sun and in the heliosphere. Space Sci. Rev. 90, 413-491 (1999). doi:10.1023/A:1005105831781

H. Rème, F. Cotin, A. Cros et al., The Giotto RPA-Copernic plasma experiment, in ESA Special Publication, vol. 1070 (1986), pp. 33-52

E. Robbrecht, D. Berghmans, R.A.M. Van der Linden, Automated LASCO CME catalog for solar cycle 23: are CMEs scale invariant? Astrophys. J. 691, 1222-1234 (2009). doi:10.1088/0004-637X/691/2/1222

D.A. Roberts, Interplanetary observational constraints on Alfvén wave acceleration of the solar wind. J. Geophys. Res. 94, 6899-6905 (1989). doi:10.1029/JA094iA06p06899

D.A. Roberts, Demonstrations that the solar wind is not accelerated by waves or turbulence. Astrophys. J. 711, 1044-1050 (2010). doi:10.1088/0004-637X/711/2/1044

H. Rosenbauer, R. Schwenn, E. Marsch et al., A survey on initial results of the HELIOS plasma experiment. J. Geophys. 42, 561-580 (1977)

M.N. Saha, The solar corona. Nature 149, 524-525 (1942). doi:10.1038/149524b0

K.H. Schatten, J.M. Wilcox, N.F. Ness, A model of interplanetary and coronal magnetic fields. Sol. Phys. 6, 442-455 (1969). doi:10.1007/BF00146478

N.A. Schwadron, D.J. McComas, Solar wind scaling law. Astrophys. J. 599, 1395-1403 (2003). doi:10.1086/ 379541

N.A. Schwadron, J. Geiss, L.A. Fisk et al., Inner source distributions: theoretical interpretation, implications, and evidence for inner source protons. J. Geophys. Res. 105, 7465-7472 (2000). doi:10.1029/ 1999JA000225

J.D. Scudder, Ion and electron suprathermal tail strengths in the transition region: support for the velocity filtration model of the corona. Astrophys. J. 427, 446-452 (1994). doi:10.1086/174155

N.R. Sheeley, Y.-M. Wang, S.H. Hawley et al., Measurements of flow speeds in the corona between 2 and $30 \mathrm{R}_{\odot}$. Astrophys. J. 484, 472-478 (1997)

S. Strong, N.-E. Raouafi, R. Decker et al., Investigating the magnitude of scattered radiation towards a spacecraft star racker in the solar probe plus orbit. Space Waether (2015, submitted)

S.T. Suess, G. Poletto, A.-H. Wang, S.T. Wu, I. Cuseri, The geometric spreading of coronal plumes and coronal holes. Sol. Phys. 180, 231-246 (1998). doi:10.1023/A:1005001618698

T.K. Suzuki, S.-I. Inutsuka, Solar winds driven by nonlinear low-frequency Alfvén waves from the photosphere: parametric study for fast/slow winds and disappearance of solar winds. J. Geophys. Res. Space Phys. 111, 6101 (2006). doi:10.1029/2005JA011502

D. Telloni, R. Bruno, L. Trenchi, Radial evolution of spectral characteristics of magnetic field fluctuations at proton scales. Astrophys. J. 805, 46 (2015). doi:10.1088/0004-637X/805/1/46

J.A. Tessein, W.H. Matthaeus, M. Wan et al., Association of suprathermal particles with coherent structures and shocks. Astrophys. J. Lett. 776, 8 (2013). doi:10.1088/2041-8205/776/1/L8

B.T. Tsurutani, E.J. Smith, D.E. Jones, Waves observed upstream of interplanetary shocks. J. Geophys. Res. 88, 5645-5656 (1983). doi:10.1029/JA088iA07p05645

C.-Y. Tu, E. Marsch, Evidence for a 'background' spectrum of solar wind turbulence in the inner heliosphere. J. Geophys. Res. 95, 4337-4341 (1990). doi:10.1029/JA095iA04p04337

B.J. Vasquez, C.W. Smith, K. Hamilton, B.T. MacBride, R.J. Leamon, Evaluation of the turbulent energy cascade rates from the upper inertial range in the solar wind at 1 AU. J. Geophys. Res. Space Phys. 112, 7101 (2007). doi:10.1029/2007JA012305

M. Velli, Solar wind acceleration: mechanisms and scaling laws, in Twelfth International Solar Wind Conference vol. 1216 (2010), pp. 14-19. doi:10.1063/1.3395823 
M. Velli, R. Grappin, A. Mangeney, Turbulent cascade of incompressible unidirectional Alfvén waves in the interplanetary medium. Phys. Rev. Lett. 63, 1807-1810 (1989). doi:10.1103/PhysRevLett.63.1807

A. Verdini, M. Velli, Alfvén waves and turbulence in the solar atmosphere and solar wind. Astrophys. J. 662, 669-676 (2007). doi:10.1086/510710

A. Verdini, M. Velli, E. Buchlin, Turbulence in the sub-Alfvénic solar wind driven by reflection of lowfrequency Alfvén waves. Astrophys. J. Lett. 700, 39-42 (2009). doi:10.1088/0004-637X/700/1/L39

A. Verdini, M. Velli, W.H. Matthaeus, S. Oughton, P. Dmitruk, A turbulence-driven model for heating and acceleration of the fast wind in coronal holes. Astrophys. J. Lett. 708, 116-120 (2010). doi:10.1088/20418205/708/2/L116

R. von Steiger, N.A. Schwadron, L.A. Fisk et al., Composition of quasi-stationary solar wind flows from Ulysses/Solar Wind Ion Composition Spectrometer. J. Geophys. Res. 105, 27217-27238 (2000). doi:10.1029/1999JA000358

A. Vourlidas, R.A. Howard, S.P. Plunkett et al., The Wide-Field Imager for Solar Probe Plus (WISPR). Space Sci. Rev. (2015). doi:10.1007/s11214-014-0114-y

Y.-M. Wang, Coronal holes and open magnetic flux. Space Sci. Rev. 144, 383-399 (2009). doi:10.1007/ s11214-008-9434-0

Y.-M. Wang, N.R. Sheeley Jr., Solar wind speed and coronal flux-tube expansion. Astrophys. J. 355, 726-732 (1990). doi:10.1086/168805

Y.-M. Wang, N.R. Sheeley Jr., Coronal plumes and their relationship to network activity. Astrophys. J. 452, 457 (1995). doi:10.1086/176317

Y.-M. Wang, N.R. Sheeley Jr., N.B. Rich, Coronal pseudostreamers. Astrophys. J. 658, 1340-1348 (2007). doi:10.1086/511416

M.E. Wiedenbeck, G.M. Mason, C.M.S. Cohen et al., Observations of solar energetic particles from ${ }^{3} \mathrm{He}$-rich events over a wide range of heliographic longitude. Astrophys. J. 762, 54 (2013). doi:10.1088/0004$637 \mathrm{X} / 762 / 1 / 54$

K. Wilhelm, Solar coronal-hole plasma densities and temperatures. Astron. Astrophys. 455, 697-708 (2006). doi:10.1051/0004-6361:20054693

Q. Xia, J.C. Perez, B.D.G. Chandran, E. Quataert, Perpendicular ion heating by reduced magnetohydrodynamic turbulence. Astrophys. J. 776, 90 (2013). doi:10.1088/0004-637X/776/2/90

M. Zhang, M.A. Lee, Stochastic acceleration of energetic particles in the heliosphere. Space Sci. Rev. 176, 133-146 (2013). doi:10.1007/s11214-011-9754-3

I. Zouganelis, M. Maksimovic, N. Meyer-Vernet, H. Lamy, K. Issautier, A transonic collisionless model of the solar wind. Astrophys. J. 606, 542-554 (2004). doi:10.1086/382866

T.H. Zurbuchen, A new view of the coupling of the sun and the heliosphere. Annu. Rev. Astron. Astrophys. 45, 297-338 (2007). doi:10.1146/annurev.astro.45.010807.154030

T.H. Zurbuchen, L.A. Fisk, G. Gloeckler, R. von Steiger, The solar wind composition throughout the solar cycle: a continuum of dynamic states. Geophys. Res. Lett. 29, 1352 (2002). doi:10.1029/2001GL013946 